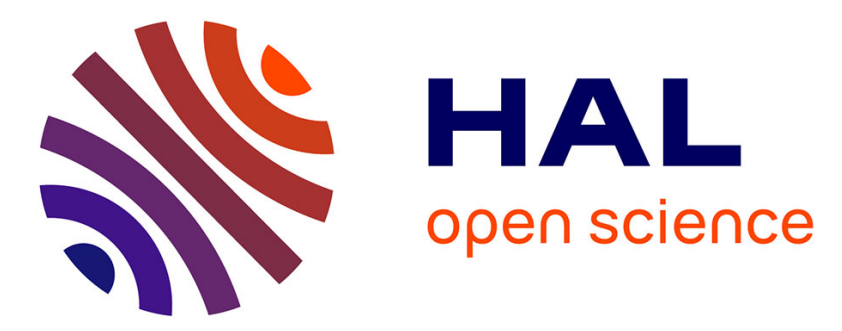

\title{
Fate of Low-Lying Charge-Transfer Excited States in a Donor:Acceptor Blend with a Large Energy Offset
}

Giacomo Londi, Saeed-Uz-Zaman Khan, Luca Muccioli, Gabriele d'Avino, Barry P Rand, David Beljonne

\section{- To cite this version:}

Giacomo Londi, Saeed-Uz-Zaman Khan, Luca Muccioli, Gabriele d'Avino, Barry P Rand, et al.. Fate of Low-Lying Charge-Transfer Excited States in a Donor:Acceptor Blend with a Large Energy Offset. Journal of Physical Chemistry Letters, 2020, 11 (23), pp.10219-10226. 10.1021/acs.jpclett.0c02858 . hal-03441999

\author{
HAL Id: hal-03441999 \\ https://hal.science/hal-03441999
}

Submitted on 26 Nov 2021

HAL is a multi-disciplinary open access archive for the deposit and dissemination of scientific research documents, whether they are published or not. The documents may come from teaching and research institutions in France or abroad, or from public or private research centers.
L'archive ouverte pluridisciplinaire HAL, est destinée au dépôt et à la diffusion de documents scientifiques de niveau recherche, publiés ou non, émanant des établissements d'enseignement et de recherche français ou étrangers, des laboratoires publics ou privés. 
On the fate of low-lying charge-transfer excited states in a donor:acceptor blend with large energy offset

Giacomo Londi ${ }^{1}$, Saeed-Uz-Zaman Khan ${ }^{2}$, Luca Muccioli ${ }^{3}$, Gabriele D’Avino ${ }^{4}$, Barry P. Rand ${ }^{2,5}$ and David Beljonne ${ }^{1 *}$

${ }^{1}$ Laboratory for Chemistry of Novel Materials, University of Mons, Place du Parc 20, 7000 Mons, Belgium

${ }^{2}$ Department of Electrical Engineering, Princeton University, Princeton, New Jersey 08544, United States

${ }^{3}$ Department of Industrial Chemistry, University of Bologna, Viale Risorgimento 4, 40136 Bologna, Italy

${ }^{4}$ Grenoble Alpes University, CNRS, Grenoble INP, Institut Néel, 25 rue des Martyrs, 38042 Grenoble, France

${ }^{5}$ Andlinger Center for Energy and the Environment, Princeton University, Princeton, New Jersey 08544, United States

*email: David.Beljonne@umons.ac.be 


\begin{abstract}
In an effort to gain a comprehensive picture for the interfacial states in bulk heterojunction solar cells, we provide a combined experimental-theoretical analysis of the energetics and dynamics of low-lying electronic charge-transfer (CT) states in donor:acceptor blends with large frontier orbital energy offset. By varying the blend composition and temperature, we unravel the static and dynamic contributions to the disordered density of states (DOS) of the CT states manifold, and assess their recombination to the ground state. Namely, we find that static disorder (conformational and electrostatic) shapes the CT DOS, and that fast non-radiative recombination crops the low-energy tail of the distribution probed by external quantum efficiency (EQE) measurements (thereby largely contributing to voltage losses). Our results then question the standard practice of extracting microscopic parameters such as exciton energy and energetic disorder from EQE.
\end{abstract}

\title{
INTRODUCTION
}

Organic solar cells (OSCs) have now reached power conversion efficiencies (PCEs) close to 18\%, with recent increases owing to the development of non-fullerene acceptors. ${ }^{1,2}$ This remarkable result has been achieved through the optimization of absorber and interface material design rules, as well as by the engineering of donor:acceptor (D:A) bulk heterojunction (BHJ) interfaces. In the quest for optimally performing materials and device architectures, the target is now on minimizing sources of photocurrent and (more importantly) voltage losses.

The most important losses in OSCs deal with the fate of tightly bound, intermolecular electron-hole (e-h) pairs, electronic states that are generated at the D:A interface upon charge-transfer (CT). ${ }^{3}$ The production of separated charges from those states requires that holes and electrons overcome their Coulomb binding energy instead of recombining to the ground state, a binding that depends on the electrostatic landscape at the interface ${ }^{4,5}$ and on the amount of the spatial delocalization of the electron and hole wavefunctions ${ }^{6}$. These CT states come with their own energy spectrum or density of states (DOS), which is typically quite broad for various reasons, including that D:A blends often comprise a heterogeneous distribution of local microstructures, and the coupling between the electronic excitations and the nuclear degrees of freedom or the involvement of multiple electron and/or hole states. Despite several investigations, the detailed origin for the DOS and the role of hot versus relaxed CT states in charge separation and recombination is still controversial..$^{7-9}$

One way to analyze the manifold of excited states is by means of external quantum efficiency (EQE) measurements, which provide a measure of the exciton DOS, weighted by the contribution of the different states to the photocurrent. ${ }^{10}$ Using this technique, we previously conducted a thorough study on D:A systems characterized by wide optical bandgaps and large frontier orbital energy offset (i.e. large energetic driving force for charge separation), resulting in ultrabroad CT state spectra. ${ }^{11}$ Typically, such combinations of materials are also characterized by a small energy offset between the donor highest occupied molecular orbital (HOMO) and the acceptor lowest unoccupied molecular orbital (LUMO), leading to an intrinsically low open circuit voltage $\left(V_{o c}\right)$, a parameter that conversely should be maximized to boost PCE. Although not at all appealing for their OSC performances, these 
systems are nevertheless a very interesting case study to resolve the existing dilemmas on the nature and fate of interfacial CT states, as these can be probed over an unusually large energy window. Here, we focus on $\mathrm{N}, \mathrm{N}^{\prime}$-di(1-naphthyl)-N,N'-diphenyl-(1,1'-biphenyl)-4,4'-diamine (NPB) : 1,4,5,8,9,11-hexaazatriphenylenehexacarbonitrile (HAT-CN) blends at different composition and temperature. In an earlier report, we discussed the existence of multiple electronic CT states at these hetero-interfaces and their contributions to the photocurrent. ${ }^{11}$ In this work, we go beyond this analysis and provide a comprehensive microscopic model for the energetics and dynamics of the first electronic CT state, disentangling the contributions from static and dynamic disorder to the DOS. Our accurate computational protocol entails a combination of molecular dynamics (MD) simulations, density functional theory (DFT) and time-dependent (TD) DFT calculations, and a microelectrostatic (ME) model ${ }^{12,13}$, specifically designed to include environmental effects (see Methods). The inclusion of both short- and long-range electrostatic effects is of particular relevance, since the effective electron affinity (EA) and ionization potential (IP) of molecules in a solid state environment and the corresponding CT excitation energies depend (often strongly) on mutual intermolecular electrostatic interactions. $^{14}$

Through this protocol, we observe that the CT DOS is broadened mostly because of conformational and electrostatic disorder associated with the NPB flexibility and the HAT-CN quadrupolar layout respectively, and is essentially static on the timescale of charge separation/recombination. We also predict fast $(\sim \mathrm{ps})$ non-radiative decay from the lowest CT states that should overcome charge separation hence rendering these tail states EQE silent. This is fully confirmed by comparison to experimental sensitive absorption versus EQE measurements showing that the CT DOS explored by EQE is cropped at low energy.

\section{RESULTS AND DISCUSSION}

Three NPB:HAT-CN blends of increasing HAT-CN molar fraction (4.6\%, 10.3\%, 76.1\%; about one thousand molecules per sample) were simulated with atomistic MD simulations, adopting a nonequilibrium protocol that reproduces vapor co-deposition. ${ }^{15-19}$ Deposition simulations were performed at $500 \mathrm{~K}$ and were followed by equilibration runs at 100 and $300 \mathrm{~K}$ (see Methods). The final structure of the sample with the highest HAT-CN molar fraction at $300 \mathrm{~K}$ is displayed in Figure 1, showing also the two components separately, in order to highlight the occurrence of microsegregation in the blend. The electrostatic potential 3D maps in Figure 1 show major differences in the charge distributions of the two molecules: HAT-CN is a flat and rigid molecule, characterized by symmetrically distributed and strongly dipolar cyano groups attached to the $\pi$ core. In contrast, NPB features a weakly polarized charge density and presents a dumbbell shape, together with a certain conformational freedom conferred by rotations about phenyl-phenyl and N-phenyl bonds (see Figure $\mathrm{S} 1$ in SI). From the differences in shape and molecular charge distribution between NPB and HAT$\mathrm{CN}$, one can surmise that their intermolecular interactions are very different, hence promoting phase separation with respect to the entropically favored full mixing, even in the solid state, and simulations show that this is actually the case. This segregation determines separate migration pathways for holes (in NPB) and electrons (in HAT-CN) from the interface to the electrodes, while still maintaining close D:A contacts. In particular, some $\pi$-stacked hetero-dimers were observed, with the HAT-CN 
azatriphenylene core adjacent and parallel to the central biphenyl moiety of NPB (see Figure S2 in SI). However, the structural analysis of the samples ruled out the presence of any particular orientational or positional order, except within $1 \mathrm{~nm}$ from the boundary with the graphene layer used as a support (not shown in Figure 1). Molecules in this region and at the very top of the sample, where there is a free surface, were therefore not included in electronic structure calculations.

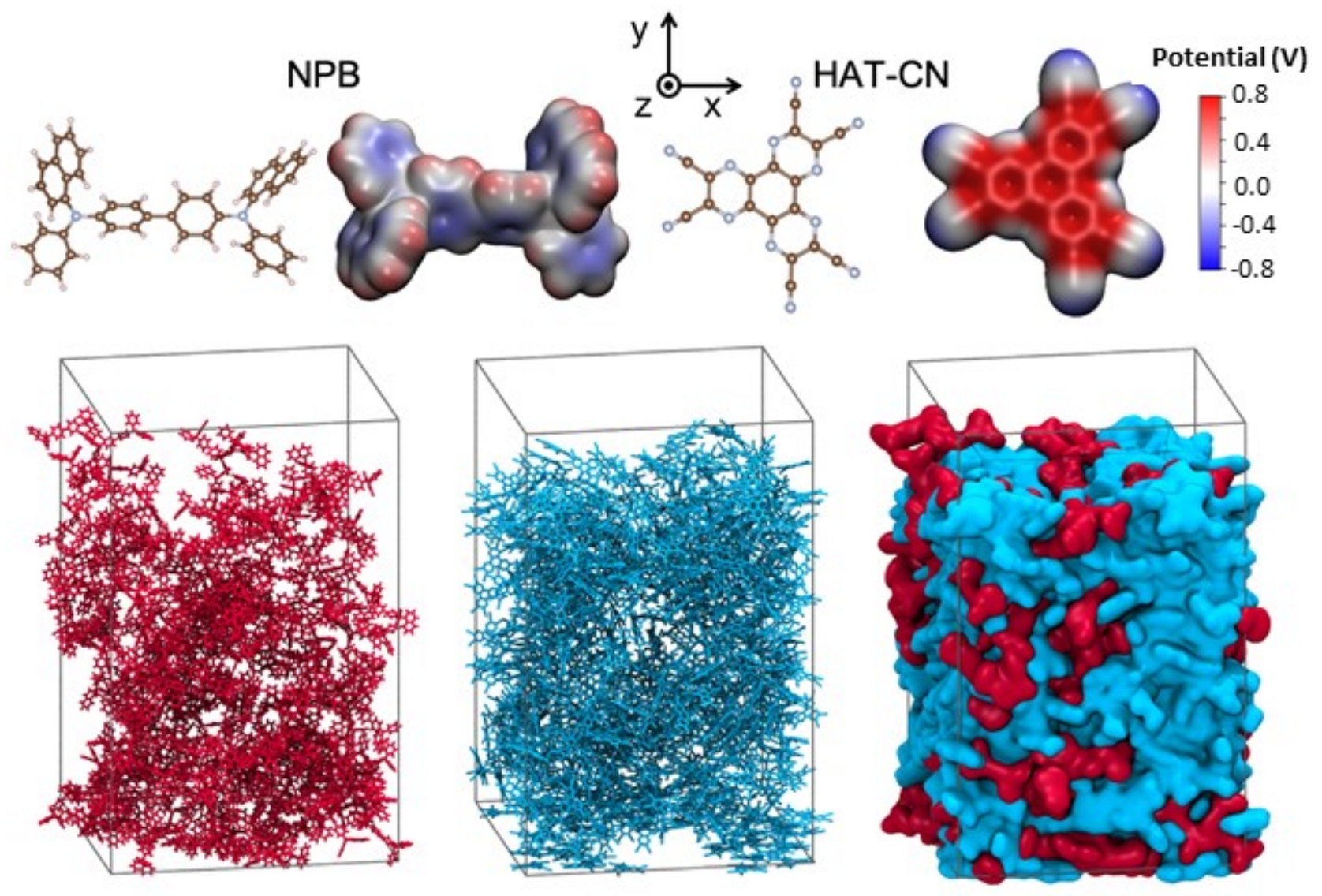

Figure 1: Top: Molecular structures and electrostatic potential color maps of N,N'-di(1-naphthyl)$\mathrm{N}, \mathrm{N}^{\prime}$-diphenyl-(1,1'-biphenyl)-4,4'-diamine (NPB) and 1,4,5,8,9,11-hexaaza triphenylene hexacarbonitrile (HAT-CN). Bottom: snapshots of the simulated sample at concentrations 24.9\% NPB:76.1\% HAT-CN. From the left to the right, visualizations of NPB molecules only (in red), HAT$\mathrm{CN}$ only (in cyan), and the whole sample with an isosurface representation.

Our account of the electronic properties in NPB:HAT-CN blends starts from the analysis of the charge transport energy levels in the solid state.

Figure 2 shows the average values (and standard deviations as error bars) of the EA of HAT-CN and the IP of NPB as a function of composition and temperature. Both IP $_{\mathrm{NPB}}$ and $\mathrm{EA}$ HAT-CN increase substantially (by up to $0.8 \mathrm{eV}$ ) and approximately linearly with the HAT-CN molar fraction, although the limited number of investigated samples does not allow to establish whether the growth is linear like in other binary molecular blends. ${ }^{20,21}$ This linearity reflects averaging of the electrostatic 
potentials

of the

two

components.

As

reported

in

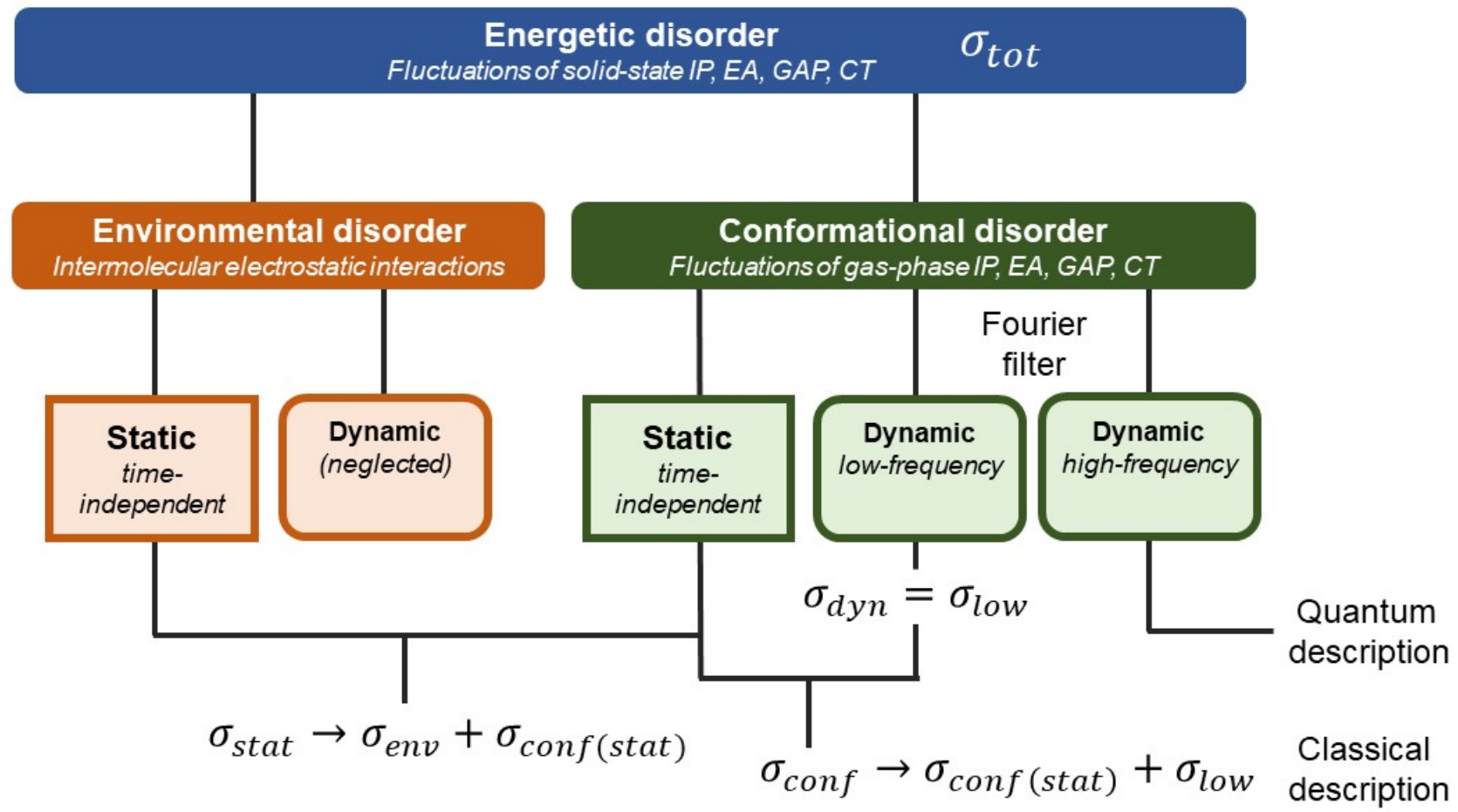

Figure 3: Logical scheme explaining the partitioning of the different contributions to the total energetic disorder $\sigma_{\text {tot }}^{\mathrm{x}}$. DFT/ME calculations were carried out in order to compute the IP of all NPB and the EA of all HAT-CN in the last MD frame of each blend. This yielded a distribution of charge carrier energy levels (IP and EA) characterized by a standard deviation $\sigma_{\text {tot }}^{\mathrm{x}}$. This contains a component related to the environmental disorder and the other related to conformational disorder $\sigma_{\text {conf }}^{\mathrm{x}}$, which can be extracted from gas-phase DFT fluctuations of IP and EA. In turn, $\sigma_{\text {conf }}^{\mathrm{x}}$ has two contributions: a static and a dynamic part. The latter $\sigma_{\text {dyn }}^{\mathrm{x}}$ is composed by intramolecular low- and high-frequency components. By applying a Fourier filter to DFT time series of molecular orbital energies, computed along the MD trajectories at $300 \mathrm{~K}$, we were able to distinguish between $\sigma_{\text {low }}^{\mathrm{x}}$ and $\sigma_{\text {high }}^{\mathrm{x}}$. The latter contribution was removed from $\sigma_{\text {conf }}^{\mathrm{x}}$, so that $\sigma_{\text {conf }}^{\mathrm{x}}$ is thus made of the static $\sigma_{\text {conf(stat) }}^{\mathrm{x}}$ and the low-frequency dynamic $\sigma_{\text {low }}^{\mathrm{x}}$ part of the conformational disorder. As regards the environmental one, we assumed $\sigma_{\text {env }}^{\mathrm{x}}$ to be static in time, neglecting its dynamic character. Therefore, within the static part $\sigma_{\text {stat }}^{\mathrm{x}}$, there are both $\sigma_{\mathrm{env}}^{\mathrm{x}}$ and $\sigma_{\text {conf(stat) }}^{\mathrm{x}}$. The same reasoning can be extended to the case where $\mathrm{x}$ stands for GAP or CT, as in Table 2.

, moving from $4.6 \%$ to $76.1 \%$ of $\mathrm{HAT}-\mathrm{CN}$ at $300 \mathrm{~K}$, the mean $\mathrm{IP}_{\mathrm{NPB}}$ increases from $6.00 \mathrm{eV}$ to 6.80 $\mathrm{eV}$, with a remarkable shift of $0.8 \mathrm{eV}$ due to the electrostatic interactions with HAT-CN, while the mean $\mathrm{EA}_{\mathrm{HAT}-\mathrm{CN}}$ increases by $0.72 \mathrm{eV}$, from 4.24 to $4.96 \mathrm{eV}$. The increase in IP and EA of the two molecules in HAT-CN-rich blends is in line with similar results on the host-dependence of energy levels ${ }^{14}$ and on zinc phthalocyanine ( $\left.\mathrm{ZnPc}\right): \mathrm{F}_{8} \mathrm{ZnPc}$ blends. ${ }^{2022}$ In our case, the electrostatic landscape is governed by the strong quadrupole moment of the HAT-CN molecule, characterized by electron 
withdrawing groups at the periphery of the $\pi$ core, which generally leads to a stabilization of occupied and empty levels.

The calculation of $\mathrm{IP}_{\mathrm{NPB}}$ and $\mathrm{EA}_{\mathrm{HAT}-\mathrm{CN}}$ also provides a first validation of the methodology, since these values can be compared with experimental data measured on vapor deposited thin films of the two pure compounds: for NPB, IPs of 5.4-5.5 eV were measured by ultraviolet photoelectron spectroscopy (UPS) ${ }^{23,24}$, while the EA of HAT-CN was reported to be larger than $4.9 \mathrm{eV}$ as probed with low-energy inverse photoemission spectroscopy (LEIPS) ${ }^{25}$, and estimated to be about $4.8 \mathrm{eV}$ by comparison between UPS measurements on NPB:HAT-CN and NPB:F $F_{4}$ TCNQ films. ${ }^{26}$ To further validate our results, it is also worth mentioning that UPS studies on mixed NPB:HAT-CN thin films indicate an increase of $\mathrm{IP}_{\mathrm{NPB}}$ at increasing HAT-CN molar fraction. ${ }^{24}$ The rather strong composition effects in

Figure 2 contrasts with the effect of temperature, hardly detectable considering the standard deviations of $\mathrm{IP}_{\mathrm{NPB}}$ and $\mathrm{EA}_{\mathrm{HAT}-\mathrm{CN}}$, suggesting a minor effect exerted by thermal fluctuations in these blends. Therefore, in the following, we will mainly focus on the results at $300 \mathrm{~K}$, while those obtained at $100 \mathrm{~K}$ are reported in Table S1 in SI. Since the shifts in the energy levels of NPB and HAT-CN are so similar, overall the photovoltaic gap defined as $\mathrm{E}_{\mathrm{GAP}}=\mathrm{IP}_{\mathrm{NPB}}-\mathrm{EA}_{\mathrm{HAT}-\mathrm{CN}}$ (see below) is not expected to change significantly with HAT-CN molar fraction.

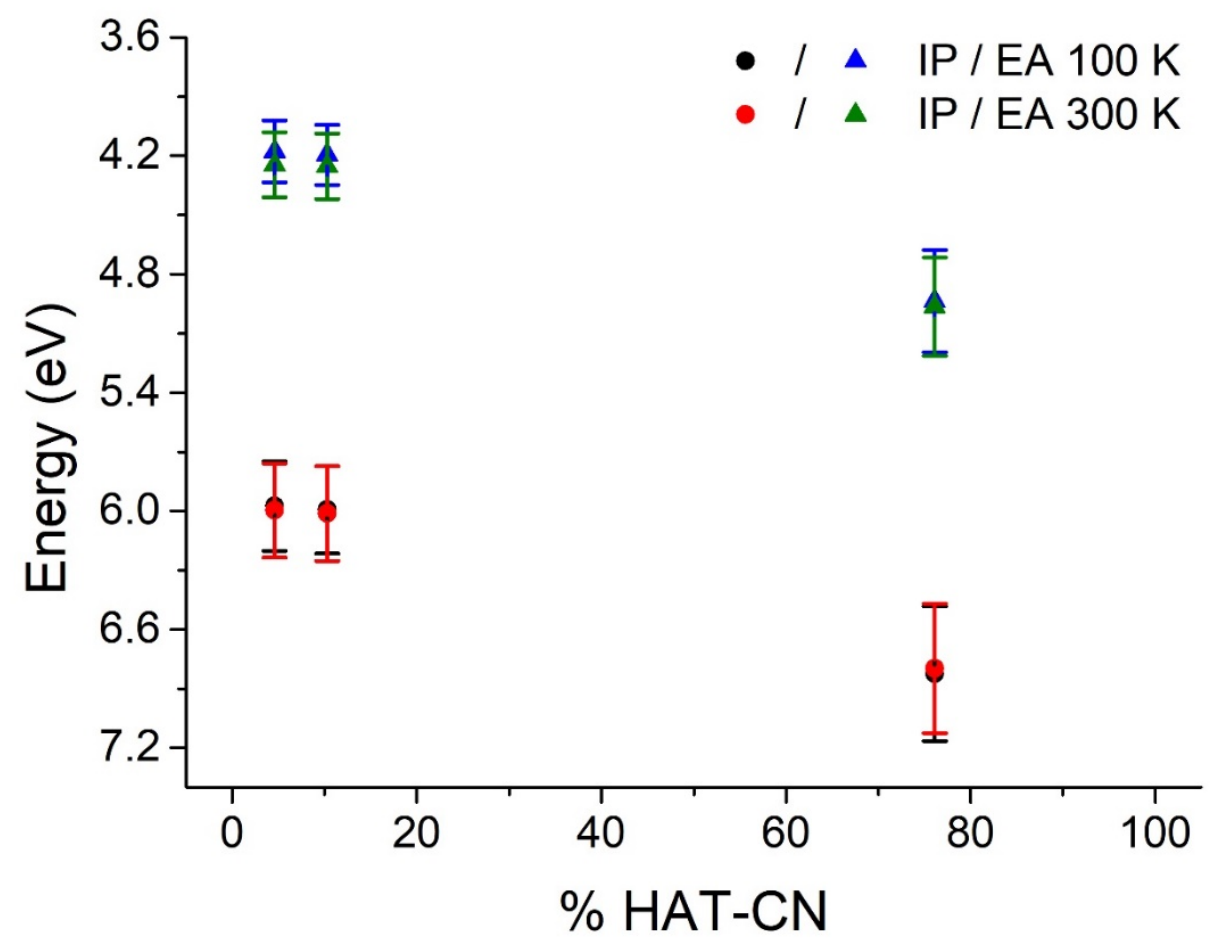

Figure 2: Average electron affinity (EA) of HAT-CN (blue and green triangles) and ionization potential (IP) of NPB (black and red circles) as a function of temperature and HAT-CN molar fraction. Vertical bars represent standard deviations. 


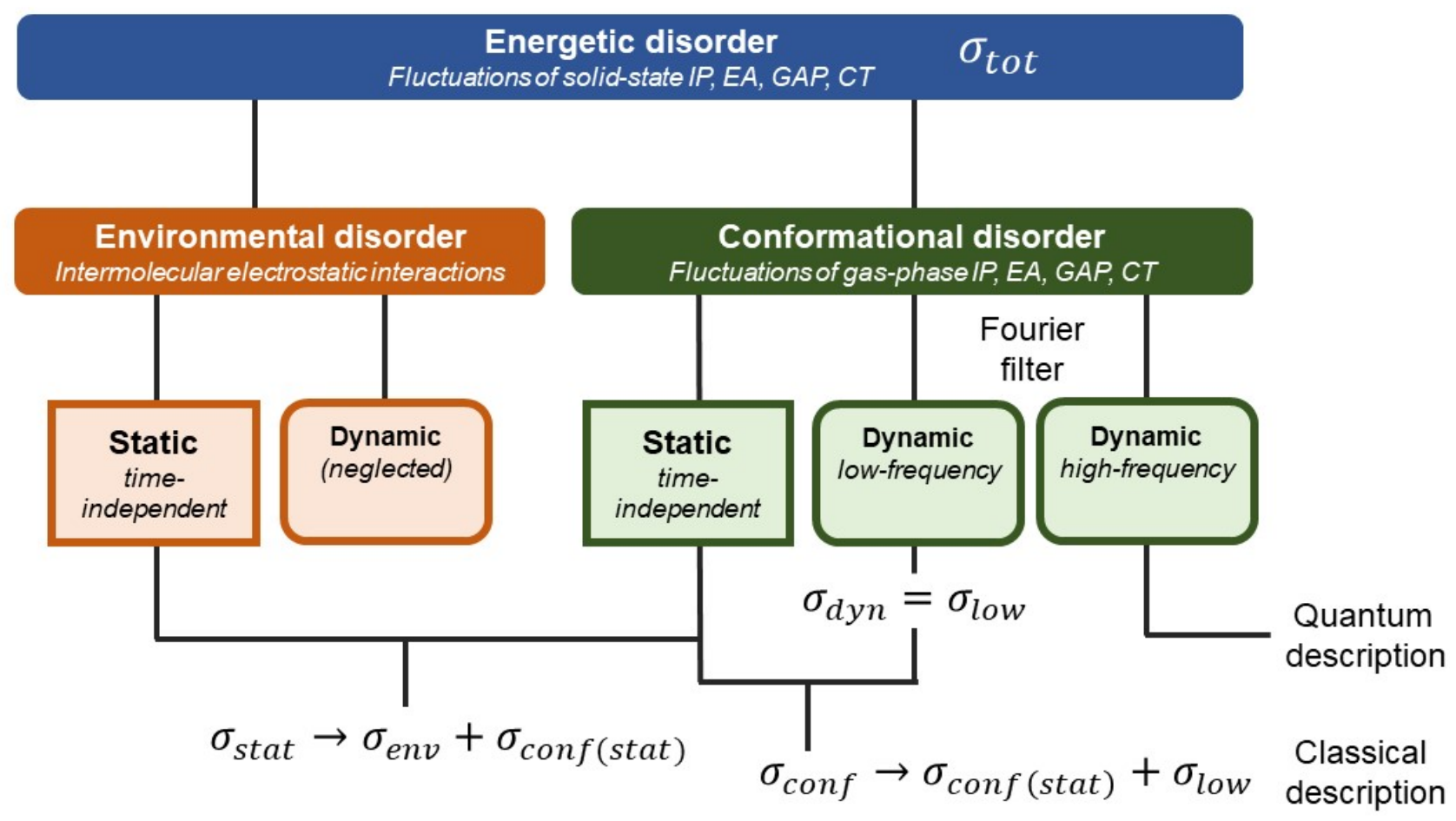

Figure 3: Logical scheme explaining the partitioning of the different contributions to the total energetic disorder $\sigma_{\text {tot. }}^{\mathrm{x}}$. DFT/ME calculations were carried out in order to compute the IP of all NPB and the EA of all HAT-CN in the last MD frame of each blend. This yielded a distribution of charge carrier energy levels (IP and EA) characterized by a standard deviation $\sigma_{\text {tot }}^{\mathrm{x}}$. This contains a component related to the environmental disorder and the other related to conformational disorder $\sigma_{\text {conf }}^{\mathrm{x}}$, which can be extracted from gas-phase DFT fluctuations of IP and EA. In turn, $\sigma_{\text {conf }}^{\mathrm{x}}$ has two contributions: a static and a dynamic part. The latter $\sigma_{\mathrm{dyn}}^{\mathrm{x}}$ is composed by intramolecular low- and high-frequency components. By applying a Fourier filter to DFT time series of molecular orbital energies, computed along the MD trajectories at $300 \mathrm{~K}$, we were able to distinguish between $\sigma_{\text {low }}^{\mathrm{x}}$ and $\sigma_{\text {high }}^{\mathrm{x}}$. The latter contribution was removed from $\sigma_{\text {conf }}^{\mathrm{x}}$, so that $\sigma_{\text {conf }}^{\mathrm{x}}$ is thus made of the static $\sigma_{\text {conf(stat) }}^{\mathrm{x}}$ and the low-frequency dynamic $\sigma_{\text {low }}^{\mathrm{x}}$ part of the conformational disorder. As regards the environmental one, we assumed $\sigma_{\text {env }}^{\mathrm{x}}$ to be static in time, neglecting its dynamic character. Therefore, within the static part $\sigma_{\text {stat }}^{\mathrm{x}}$, there are both $\sigma_{\mathrm{env}}^{\mathrm{x}}$ and $\sigma_{\text {conf(stat) }}^{\mathrm{x}}$. The same reasoning can be extended to the case where $\mathrm{x}$ stands for GAP or CT, as in Table 2.

Having established the dependence on composition and temperature of the average IP and EA, we now focus on energetic disorder, i.e. the spread of energy levels. In order to provide a deeper insight, 


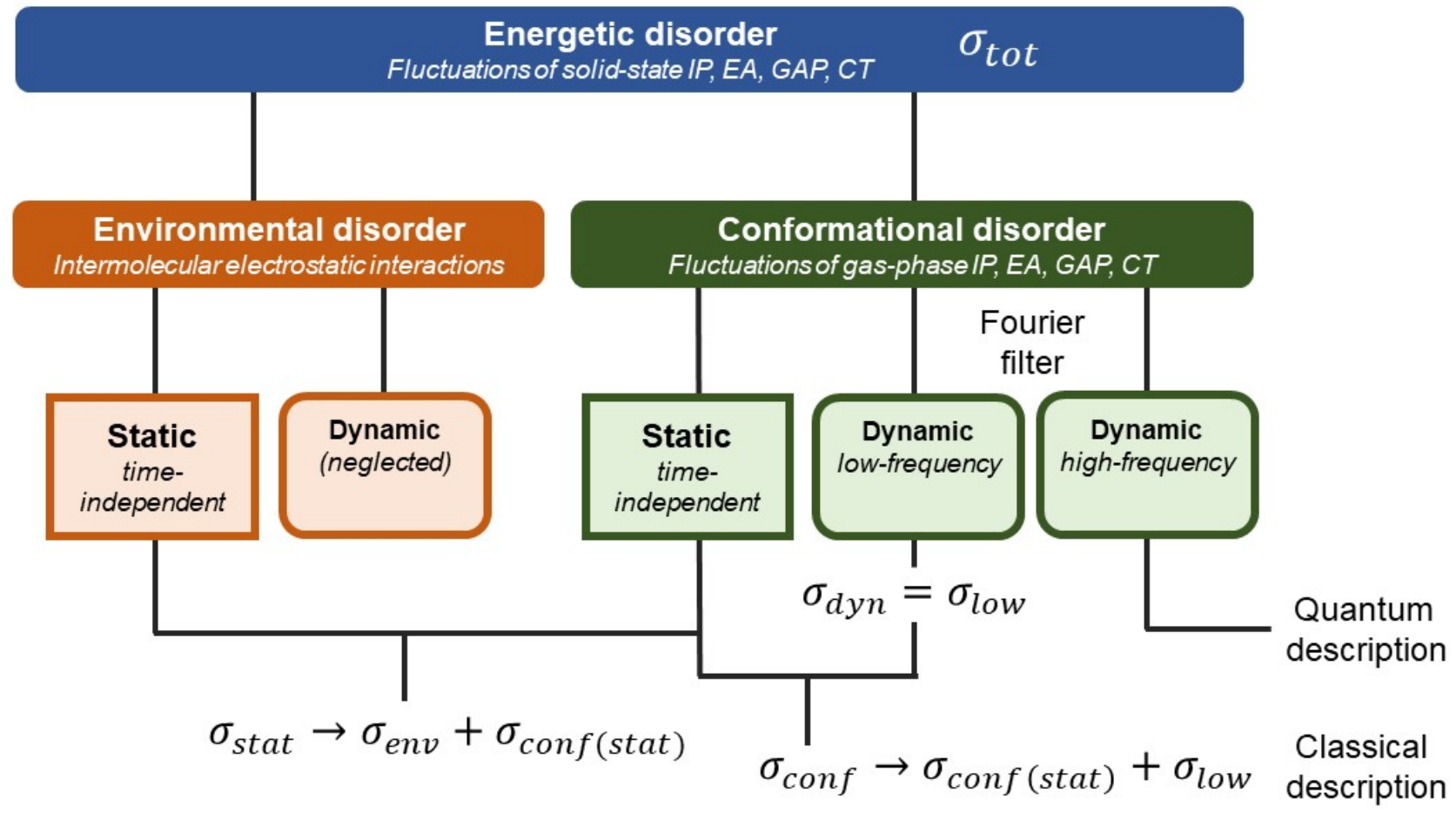

Figure 3: Logical scheme explaining the partitioning of the different contributions to the total energetic disorder $\sigma_{\text {tot }}^{\mathrm{x}}$. DFT/ME calculations were carried out in order to compute the IP of all NPB and the EA of all HAT-CN in the last MD frame of each blend. This yielded a distribution of charge carrier energy levels (IP and EA) characterized by a standard deviation $\sigma_{\text {tot }}^{\mathrm{x}}$. This contains a component related to the environmental disorder and the other related to conformational disorder $\sigma_{\text {conf }}^{\mathrm{x}}$, which can be extracted from gas-phase DFT fluctuations of IP and EA. In turn, $\sigma_{\text {conf }}^{\mathrm{x}}$ has two contributions: a static and a dynamic part. The latter $\sigma_{\text {dyn }}^{\mathrm{x}}$ is composed by intramolecular low- and high-frequency components. By applying a Fourier filter to DFT time series of molecular orbital energies, computed along the MD trajectories at $300 \mathrm{~K}$, we were able to distinguish between $\sigma_{\text {low }}^{\mathrm{x}}$ and $\sigma_{\text {high }}^{\mathrm{x}}$. The latter contribution was removed from $\sigma_{\text {conf }}^{\mathrm{x}}$, so that $\sigma_{\text {conf }}^{\mathrm{x}}$ is thus made of the static $\sigma_{\text {conf(stat) }}^{\mathrm{x}}$ and the low-frequency dynamic $\sigma_{\text {low }}^{\mathrm{x}}$ part of the conformational disorder. As regards the environmental one, we assumed $\sigma_{\text {env }}^{\mathrm{x}}$ to be static in time, neglecting its dynamic character. Therefore, within the static part $\sigma_{\text {stat }}^{\mathrm{x}}$, there are both $\sigma_{\mathrm{env}}^{\mathrm{x}}$ and $\sigma_{\text {conf(stat) }}^{\mathrm{x}}$. The same reasoning can be extended to the case where $\mathrm{x}$ stands for GAP or CT, as in Table 2.

dissect the total energetic disorder obtained from our atomistic simulations (standard deviation $\sigma_{\text {tot }}^{x}$, where $\mathrm{x}$ stands for IP or EA) into different contributions, as depicted in Figure 3. Specifically, energetic disorder can be characterized in terms of its physical origin, and here we distinguish between conformational $\left(\sigma_{\text {conf }}^{x}\right.$, an intramolecular contribution related to different molecular geometries sampled in MD simulations) and environmental disorder ( $\sigma_{e n v}^{x}$, due to intermolecular electrostatic interactions). Energetic disorder can also be classified according to timescales as being of static $\left(\sigma_{\text {stat }}^{x}\right)$ or dynamic $\left(\sigma_{\text {low }}^{x}\right)$ nature. While static disorder is mostly sourced by inhomogeneity 
in the electrostatic environment of the CT pairs, dynamic disorder includes contributions from intramolecular low-frequency modes (below $200 \mathrm{~cm}^{-1}$ ) that are correctly described by our classical MD simulations in the temperature range explored. We note that, unlike recent studies addressing dynamic disorder in disordered molecular blends with multilevel computational approaches ${ }^{27-29}$, the IP and EA fluctuations due to high-frequency vibrations were filtered out in the calculation of energetic disorder, which hence accounts only for low-frequency classical modes (see Methods). The contribution from high-frequency vibrations was then included in a proper quantum-mechanical framework in the calculation of the absorption spectrum and non-radiative recombination rates (Equations 3 and 5).

Table 1: Average energy of single carrier transport levels (in eV) and standard deviations $\sigma_{\text {tot }}^{x}$ (in $\mathrm{meV}$, where $\mathrm{x}$ stands for IP or EA) in NPB:HAT-CN blends at $300 \mathrm{~K}$. Energy fluctuations (in meV) were also quantified according to their nature: intramolecular $\sigma_{\text {conf }}^{x}$, intermolecular $\sigma_{\text {env }}^{x}$, static $\sigma_{\text {stat }}^{x}$ and low-frequency dynamic disorder $\sigma_{\text {low }}^{x}$.

\begin{tabular}{|c|c|c|c|c|c|c|}
\hline \% HAT-CN & $\mathrm{IP}_{\mathrm{NPB}}$ & $\sigma_{\text {tot }}^{I P}$ & $\sigma_{\text {conf }}^{I P}$ & $\sigma_{\text {env }}^{I P}$ & $\sigma_{\text {stat }}^{I P}$ & $\sigma_{\text {low }}^{I P}$ \\
\hline 4.6 & 6.00 & 239 & 179 & 161 & 223 & 87 \\
\hline 10.3 & 6.01 & 241 & 188 & 164 & 222 & 94 \\
\hline 76.1 & 6.80 & 328 & 183 & 274 & 311 & 102 \\
\hline
\end{tabular}

\begin{tabular}{|c|c|c|c|c|c|c|}
\hline$\%$ HAT-CN & EA $_{\mathrm{HAT}-\mathrm{CN}}$ & $\sigma_{\text {tot }}^{\text {EA }}$ & $\sigma_{\text {conf }}^{\text {EA }}$ & $\sigma_{\text {env }}^{\text {EA }}$ & $\sigma_{\text {stat }}^{\text {EA }}$ & $\sigma_{\text {low }}^{\text {EA }}$ \\
\hline 4.6 & 4.24 & 165 & 57 & 151 & 164 & 19 \\
\hline 10.3 & 4.25 & 166 & 47 & 155 & 165 & 19 \\
\hline 76.1 & 4.96 & 249 & 42 & 244 & 249 & 19 \\
\hline
\end{tabular}

Environmental effects due to long-range intermolecular electrostatic interactions represent the predominant contribution to the total energetic disorder: in amorphous blends each molecule experiences a different electrostatic environment that leads to a large spread in the energy levels. The environmental disorder $\sigma_{e n v}^{x}$ comparably affects IP $\mathrm{NPB}_{\mathrm{NPB}}$ and EAHAT-CN and markedly increases with the HAT-CN molar fraction, revealing that the main source of electrostatic disorder is indeed the strongly polarized charge density of the acceptor molecule (see Figure 1). The conformational disorder $\sigma_{\text {conf }}^{x}$ is, instead, only weakly sensitive to composition, but different between the two molecules. The calculated $\sigma_{\text {conf }}^{x}$ are indeed much larger for NPB, owing to the flexible molecular structure of the donor molecule, characterized by soft torsional degrees of freedom, as compared to the rather rigid HAT-CN acceptor. This dissimilarity between the two components is even more pronounced when looking at the conformational low-frequency dynamic disorder $\sigma_{\text {low }}^{x}$, which is the sole contribution with a clear temperature dependence, in a way compatible with that prescribed for coupled classical harmonic oscillators. In summary, electrostatic and conformational fluctuations similarly contribute to the energetic disorder in $\mathrm{IP}_{\mathrm{NPB}}$, while the spread in EAHAT-CN is essentially determined by the former. 

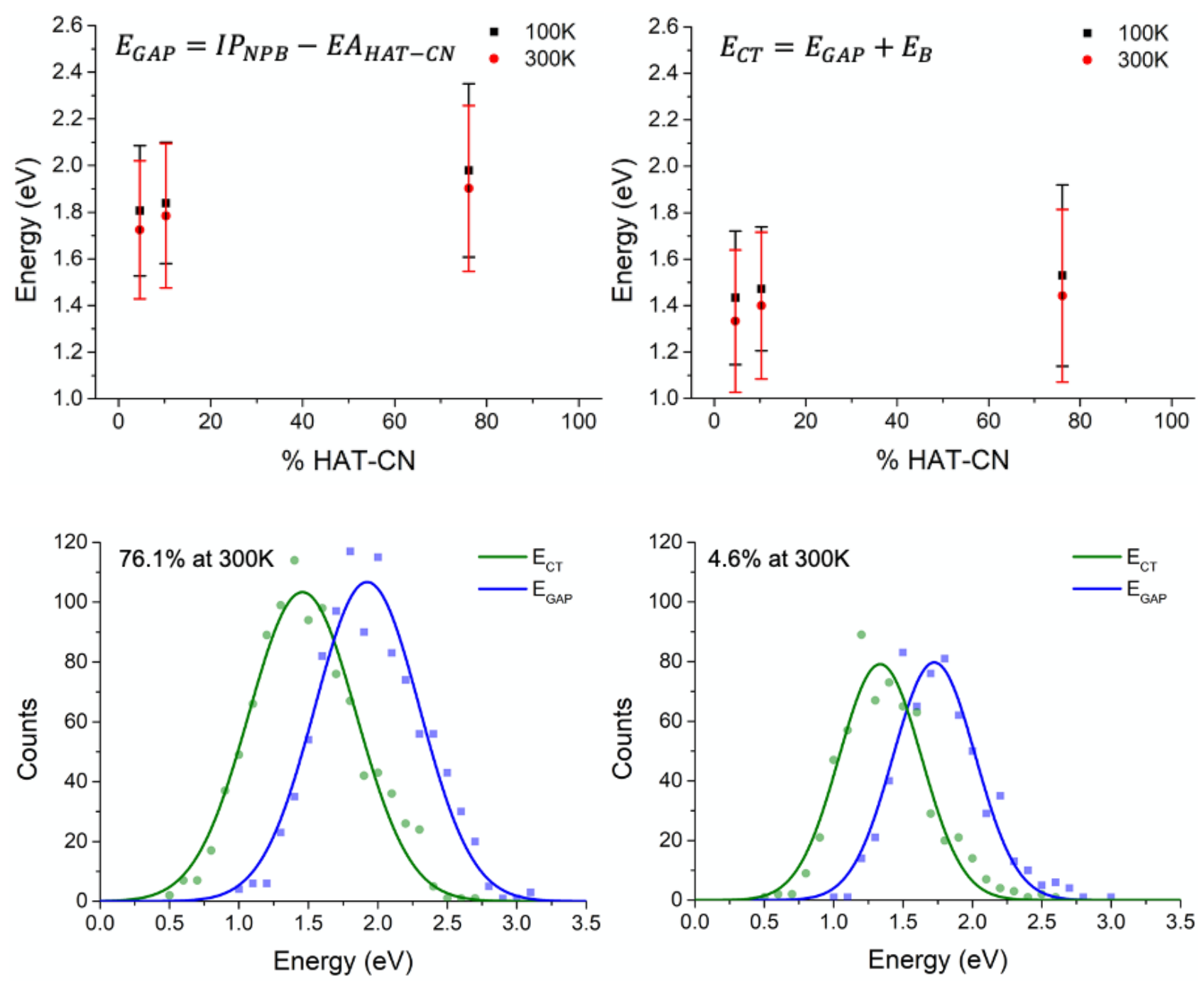

Figure 4: Top: Average photovoltaic gap $\left(\mathrm{E}_{\mathrm{GAP}}\right)$ and $\mathrm{CT}$ state energies $\left(\mathrm{E}_{\mathrm{CT}}\right)$ for NPB:HAT-CN pairs as a function of HAT-CN molar fraction and temperature. Vertical bars represent standard deviations. Bottom: CT density of states (DOS) and distribution of gap energies at two different HAT-CN molar fractions at $300 \mathrm{~K}$. Continuous lines are Gaussian fits.

The analysis of CT excitations, whose statistics was gathered on NPB:HAT-CN dimers in our MDsimulated samples, is presented in Figure 4 and Table 2 (see also Table S2 and Figure S4 in SI for the data at $100 \mathrm{~K}$ ). As anticipated in the discussion of the transport levels, notwithstanding their large variations with the blend composition, the mean photovoltaic gap $\mathrm{E}_{\mathrm{GAP}}=\mathrm{IP}_{\mathrm{NPB}}-\mathrm{EA}_{\mathrm{HAT}-\mathrm{CN}}$ changes by less than $0.2 \mathrm{eV}$, going from $1.72 \mathrm{eV}$ at $4.6 \%$ to $1.90 \mathrm{eV}$ at $76.1 \%$ of HAT-CN. The mean energy of the first $\mathrm{CT}$ state, $\mathrm{E}_{\mathrm{CT}}$, shows even smaller variations with composition. The exciton binding energy, $\mathrm{E}_{\mathrm{B}}=\mathrm{E}_{\mathrm{CT}}-\mathrm{E}_{\mathrm{GAP}}$, is predicted to be about $-0.4 \mathrm{eV}$, in line with typical values expected in organic semiconductors. ${ }^{30}$ The large standard deviation $\sigma_{\text {tot }}^{C T}$, reaching $371 \mathrm{meV}$ at high HAT-CN molar fraction, is dominated by the fluctuations in the photovoltaic gap, rather than by those in the binding energy. It is largely due to the rough electrostatic landscape produced by HAT-CN molecules and to the conformational disorder of flexible NPBs, both contributions being essentially static in 
time. The broad distribution of CT states energies observed experimentally in EQE spectra (see below) could be hence imputable to the energetic disorder in the hole and electron energy levels.

Table 2: Average photovoltaic gap $\left(E_{G A P}\right), \mathrm{CT}$ state $\left(E_{C T}\right)$, exciton binding energies $\left(E_{B}\right)($ in eV) and standard deviations $\sigma_{\text {tot }}^{x}$ (in meV, where x stands for GAP or CT) for NPB:HAT-CN pairs at $300 \mathrm{~K}$ as a function of HAT-CN molar fraction. Energy fluctuations (in $\mathrm{meV}$ ) were also quantified according to their nature: intramolecular $\sigma_{\text {conf }}^{x}$, intermolecular $\sigma_{\text {env }}^{x}$, static $\sigma_{\text {stat }}^{x}$ and low-frequency dynamic disorder $\sigma_{\text {low }}^{x}$. Environmental reorganization energies $\lambda_{e n v}(\mathrm{in} \mathrm{meV})$ were calculated as $\lambda_{\text {env }}=\frac{\sigma_{\text {low }}^{x}}{2 k_{B} T}$. Note that for photovoltaic gaps and CT state energies the intermolecular $\sigma_{e n v}^{x}$ and low-frequency dynamic disorder $\sigma_{\text {low }}^{x}$ coincide and thus are given just once.

\begin{tabular}{|c|c|c|c|c|c|c|c|}
\hline$\%$ HAT-CN & $\mathrm{E}_{\mathrm{GAP}}$ & $\sigma_{\text {tot }}^{\text {GAP }}$ & $\sigma_{\text {conf }}^{\text {GAP }}$ & $\sigma_{\text {env }}^{\text {GAP }}$ & $\sigma_{\text {stat }}^{\text {GAP }}$ & $\sigma_{\text {low }}^{\text {GAP }}$ & $\lambda_{\text {env }}$ \\
\hline 4.6 & 1.72 & 296 & 184 & 238 & 282 & 89 & 155 \\
\hline 10.3 & 1.78 & 310 & 191 & 239 & 295 & 96 & 181 \\
\hline 76.1 & 1.90 & 355 & 184 & 339 & 340 & 104 & 209 \\
\hline
\end{tabular}

\begin{tabular}{|c|c|c|c|c|c|}
\hline \% HAT-CN & $\mathrm{E}_{\mathrm{CT}}$ & $\sigma_{\text {tot }}^{C T}$ & $\sigma_{\text {stat }}^{C T}$ & $\mathrm{E}_{\mathrm{B}}$ & $\sigma_{B}$ \\
\hline 4.6 & 1.33 & 306 & 293 & -0.39 & 123 \\
\hline 10.3 & 1.40 & 316 & 301 & -0.38 & 118 \\
\hline 76.1 & 1.44 & 371 & 357 & -0.46 & 133 \\
\hline
\end{tabular}

Is also worth inspecting the DOSs plotted in Figure 4, and in particular the data at low HAT-CN molar fraction, recalling that, owing to its six cyano groups with strong electron-withdrawing character, HAT-CN was proposed and tested as a p-type dopant for organic semiconductors, ${ }^{31}$ although its EA is somewhat lower than effective p-dopants like 7,7,8,8-tetracyano-2,3,5,6tetrafluoroquinodimethane $\left(\mathrm{F}_{4} \mathrm{TCNQ}\right) .{ }^{31}$ From an energetic standpoint, doping would require $\mathrm{E}_{\mathrm{CT}}$ values as low as possible, with a sizeable part of the tail of the CT DOS extending down to negative energies. This requirement is not met in Figure 3 in line with studies of NPB:HAT-CN based lightemitting diodes (also confirming the results reported in Zhang et $a l .{ }^{32}$ ), although doping cannot be ruled out at very high HAT-CN concentrations.

The existence of an important contribution from static, temperature-independent, disorder in the real system is confirmed by the fits of low-energy portion of the EQE spectra at different temperatures. Experimental EQE spectra of a NPB:HAT-CN BHJ solar cell with $66.0 \%$ of HAT-CN are shown in Figure $5 \mathrm{a}$ for temperatures ranging from $110 \mathrm{~K}$ to $296 \mathrm{~K}$. With decreasing temperature, the EQE steadily decreases as the generation and transport of charge carriers become increasingly difficult. ${ }^{33,34}$ Despite the decrease in magnitude, the EQE spectral shape is mostly preserved but has a small redshift of the lowest energy CT peak with decreasing temperature. Following Burke et al. ${ }^{35}$, we performed a fit of EQE spectra to extract the static and dynamic contributions to the total energetic disorder as a function of temperature (see Methods). As shown in Figure $5 \mathrm{~b}$, the linear extrapolation of $\sigma_{E Q E}^{2}$ to $0 \mathrm{~K}$ provides an estimate for the static disorder of $150 \mathrm{meV}$ (see Table S3 in SI). Therefore, static 
disorder dominates the $\mathrm{CT}$ spectral linewidth even at room temperature, quite contrary to observations on most fullerene-based archetypal OSCs ${ }^{36}$, but qualitatively in agreement with the theoretical data presented in Table 2 for NPB:HAT-CN blends.
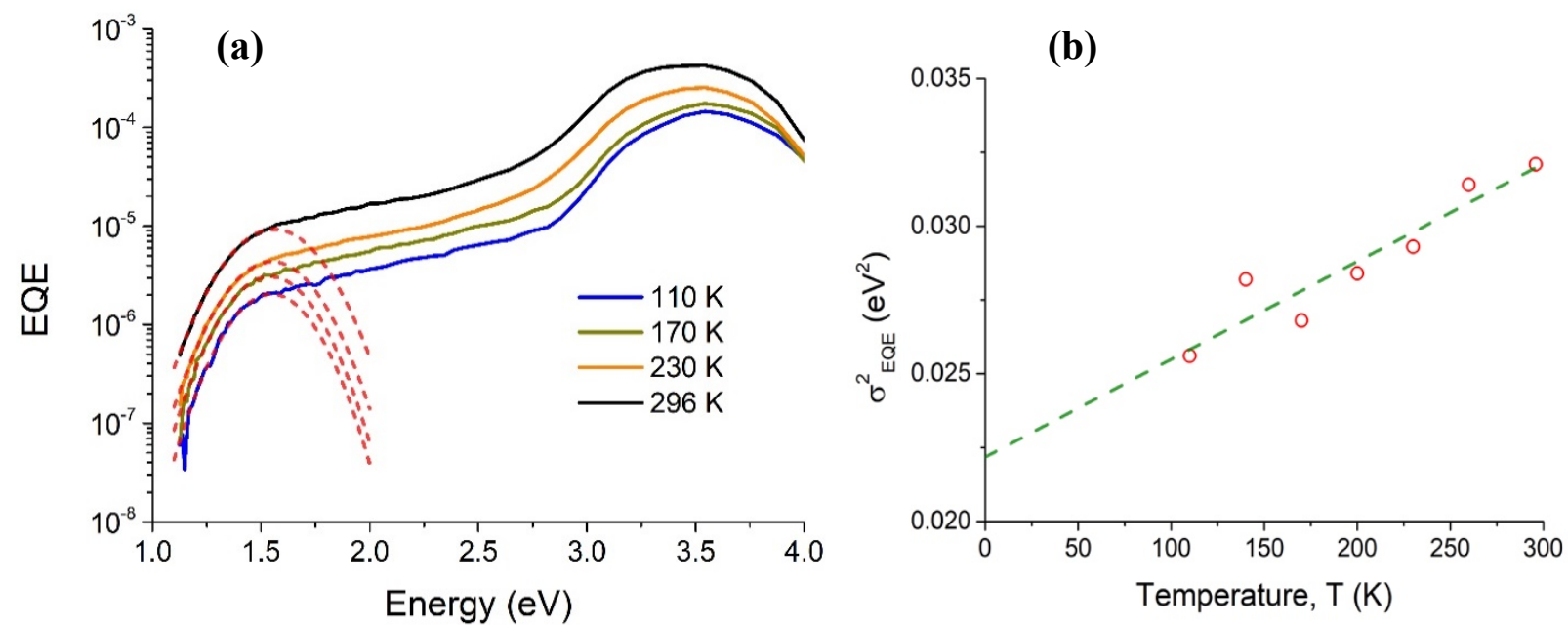

Figure 5: (a) Temperature-dependent external quantum efficiency (EQE) spectra of a NPB:HAT-CN BHJ solar cell with $66.0 \%$ of HAT-CN (solid lines). Lowest-energy CT spectra were fitted, following Burke et al.'s approach ${ }^{35}$, considering a Gaussian CT energetic distribution (dashed red lines). (b) Temperature dependence of the variance of the energetic disorder extracted from a Gaussian fit of EQE spectra. The extrapolation to $0 \mathrm{~K}$ (dashed green line) allows to estimate a static, temperatureindependent, disorder of $150 \mathrm{meV}$.

A quantitative comparison between the calculated and the EQE-derived disorder requires some caution, since the EQE spectral shape may be severely affected by charge recombination. A first hint that the EQE spectrum is not able to fully capture the CT DOS is provided by the striking difference with the absorption (photothermal deflection spectroscopy, PDS) spectrum, shown in Figure 6a. The comparison between the two spectra reveals that the lowest-energy transition, peaking at around 1.2 $\mathrm{eV}$ in the PDS, is almost absent in the EQE. In order to rationalize this dissimilarity, we exploit the in-depth knowledge of the CT DOS from our calculations to consider non-radiative recombination, an intrinsic process causing severe voltage losses in organic photovoltaic devices. ${ }^{7,37}$ Within the Marcus-Levich-Jortner framework, non-radiative recombination rates $\left(\kappa_{N R A D}\right)$ were computed and their average values binned over small $(100 \mathrm{meV})$ energy intervals are shown in Figure 6a (the full data set is given in Figure S5 and S6 in SI). Irrespective of the blend composition, the rates exceed $10^{12} \mathrm{~s}^{-1}$ for CT energies below $1.5 \mathrm{eV}$ and decrease by several orders of magnitude at increasing CT energies, a trend that is reminiscent of the so-called energy-gap law. ${ }^{38,39}$ Such large rates for the lowest-energy CT states suggest that these excitations strongly recombine to the ground state before charge dissociation can occur, explaining the difference in shape of the measured EQE and absorption spectra. Thus, unlike higher-energy CT or Frenkel excitations, the lowest-energy CT states are photovoltaically inactive in NPB:HAT-CN blends, viz. they do not contribute to photocurrent generation, and may be responsible for large voltage losses. ${ }^{37,39}$ 
The analysis above allows us to conclude that the EQE spectra are not fully representative of the actual CT DOS, at least in large offset, low gap, D:A blends, as non-radiative processes tend to crop the low-energy tails of the probed distributions. As such, the results of common Gaussian fits of the tail of EQE spectra to extract the band maximum and width should be taken with caution. Note that the EQE fit in Figure 6a seems to yield a peak position that is $\sim 0.7 \mathrm{eV}$ apart from the first absorption peak, hence the associated standard deviation cannot be interpreted as a measure of the energetic disorder in the lowest-energy CT state. The next question that arises is whether optical absorption, electroluminescence (EL) or photoluminescence (PL) measurements provide a more representative proxy for the DOS of the system, and hence would be more suited to quantify the total energetic disorder. Figure $6 \mathrm{~b}$ compares the experimental and calculated absorption spectra and the calculated CT DOS. The last two quantities were computed taking into account the coupling to an effective quantum vibrational mode, in order to describe the broadening due to vibronic progression and allow for a fair comparison with the experimental absorption (see Methods). The calculated absorption matches well with experiment, capturing the peak position, yet overestimating the bandwidth. Moreover, calculations show that the absorption spectrum gives a close reproduction of the lowenergy tail of the CT DOS, since transition dipole moments do not feature orders of magnitude variations in the energy range of interest. These results suggest that absorption spectra, although more challenging to collect experimentally for weakly-absorbing intermolecular excitons, can provide a faithful description of the CT DOS in D:A blends, and independent of the spectral region (i.e. infrared versus visible). In contrast, emission spectra suffer from the competition with non-radiative processes as does EQE and should thus be interpreted equally with care.

(a)

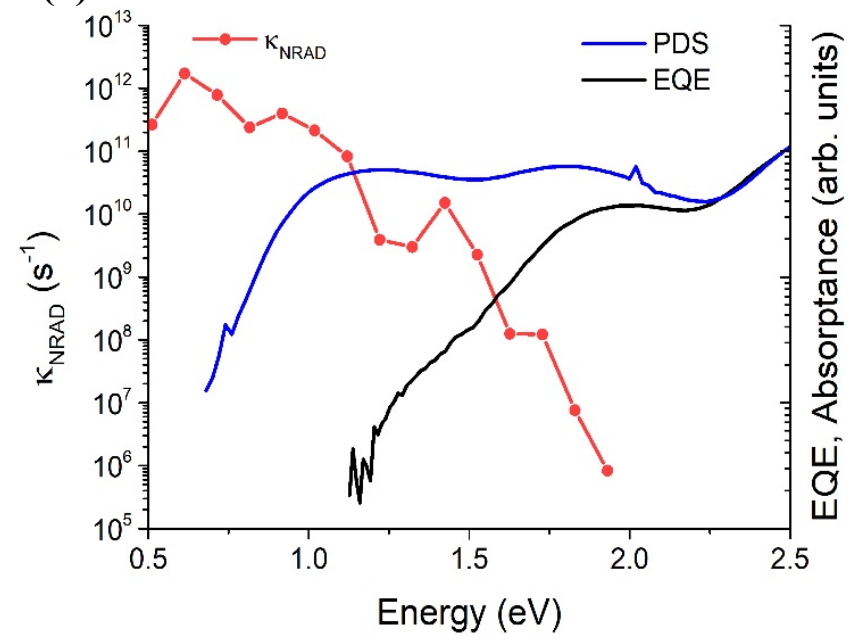

(b)

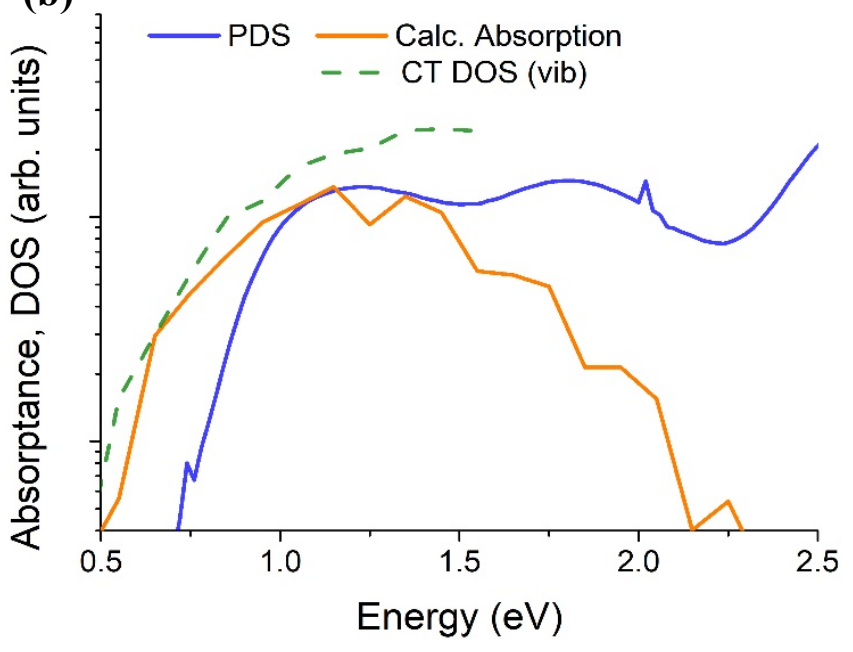

Figure 6: (a) External quantum efficiency (EQE) spectrum (solid black line) and absorptance spectrum (solid blue line) of a NPB:HAT-CN film measured by photothermal deflection spectroscopy $(\mathrm{PDS})^{11}$. Red points show the average calculated non-radiative decay rates of CT excitations to the ground state as a function of the excitations energy. The six orders of magnitude drop of $\kappa_{N R A D}$ in the CT states energy window rationalizes the stark difference between EQE and PDS spectra. (b) PDS spectrum compared to the calculated one (solid orange line) and to the calculated vibrationally- 
broadened CT density of states (DOS) (dashed green line). Experimental and calculated data refer to samples with $4.6 \%$ of HAT-CN at $300 \mathrm{~K}$.

The origin for the $\mathrm{CT}$ energetic disorder in organic photovoltaic systems was recently debated in the literature. ${ }^{7-9}$ Specifically, different conclusions regarding the relative contributions from static versus dynamic (thermally activated) disorder were reached in research papers on D:A blends based on fullerene derivatives. Comparable static and dynamic disorder was found for polymer: phenyl-C61butyric acid methyl ester (PCBM) blends, ${ }^{40}$ while a picture solely based on thermally activated dynamic disorder due to low-frequency intramolecular mode seemed to be able to correctly describe small molecule- $\mathrm{C}_{60}$ systems. ${ }^{33}$ For the latter family of systems, the evidence for a temperatureindependent energetic disorder was very recently ascribed to the zero-point motion of quantum vibrational modes. ${ }^{9}$ Besides raising some concerns on the experimental determination of the disorder, our microscopic analysis suggests that the answer to the paramount question regarding the nature of the disorder depends markedly on the system investigated and, most importantly, on the way it is probed. If, on one hand, it is plausible that the static environmental disorder is almost negligible for blends of donor molecules diluted in electrically neutral fullerenes, this contribution can be substantial in the presence of molecules featuring polar groups, such as the NPB:HAT-CN system investigated here, and whose broad CT state spectral signature is mostly due to static disorder (both conformational and electrostatic), with temperature-dependent dynamic disorder having a much lower impact.

\section{CONCLUSIONS}

The nature of low-lying CT states in NPB:HAT-CN amorphous D:A blends was investigated with a multiscale computational approach. We found that the rather wide CT DOS broadening is dominated by static disorder and arises from both conformational and electrostatic landscape variations, which in turn originate from NPB flexibility and HAT-CN quadrupoles. The large broadening and the deep LUMO levels of HAT-CN ensure that a large portion of the CT DOS lies below $1.5 \mathrm{eV}$, where nonradiative decay to the ground state becomes predominant over charge separation. This energetic situation has two important consequences, sometimes not adequately considered in the literature: i) the tail of the CT DOS cannot be probed via EQE, EL, or PL measurements, and more importantly for OSC applications, it does not contribute to charge generation but rather to recombination; ii) in such a case, the standard procedure of fitting the tail of the EQE signal with a Gaussian function to extract static and dynamic disorder becomes unreliable.

\section{METHODS}

Computational details Three different films of NPB and HAT-CN at different relative concentrations were prepared by vapor co-deposition on graphene, by means of NVT non-equilibrium MD simulations, ${ }^{15,16,18}$ for a final film thickness exceeding in all cases $10 \mathrm{~nm}$. All simulations were performed with the NAMD code. ${ }^{41}$ Systems were modelled for OLED materials, ${ }^{42}$ which allows for 
an integration timestep of $2 \mathrm{fs}$. Charges of the united atoms were obtained by fitting the electrostatic potential (ESP charges) calculated at the optimized PBE0/def2-TZVP geometry. Concerning the validation of the force field (FF), in Ref. ${ }^{42}$, where the FF parameters were optimized for reproducing the experimental crystal cells and densities of several polyaromatic compounds, NPD was taken as a test molecule outside the training set, giving an overall error of $1.1 \%$. For HATCN, we obtained a lower but reasonable agreement, by means of NVT and NPT simulations at fixed cell angles using the same equilibration protocol discussed in Ref.42, between the calculated cell dimensions $\mathrm{a}=\mathrm{b}=23.464 \AA, \mathrm{c}=15.75 \AA$, and the experimental ones $\mathrm{a}=\mathrm{b}=23.637 \AA, \mathrm{c}=14.83 \AA^{43}$. A horizontal graphene surface of $76.136 \AA \times 76.572 \AA$ was chosen as a simple rigid and chemically compatible support and kept fixed during all the simulations. Parameters for graphene-molecule interactions were obtained using Steele's potential ${ }^{44}$ and Lorentz-Berthelot mixing rules. The vertical side of the simulation box was set to $400 \AA$ to leave ample space for deposited molecules and avoid interactions between the two sides of the graphene + blend slab, since 3D periodic boundary conditions were used. Deposition proceeded by randomly selecting the chemical species of the new molecule to be added at each step according to the desired molar fraction. Every new molecule was placed at about $20 \AA$ above the forming organic layer, endowed with a velocity of $1 \AA \mathrm{ps}^{-1}$ directed parallelly to the normal to the graphene surface, and the dynamic of the system evolved for $100 \mathrm{ps}$ at $500 \mathrm{~K}$. At the end of this deposition step, if the last deposited molecule had desorbed, the step was repeated without randomizing again the molecular species, otherwise a further molecule was inserted following the same scheme. As a consequence of the randomness of the deposition, the final compositions do not match exactly the mol:mol ratio given in input (NPB:HAT-CN $=1: 3,9: 1,29: 1$ ). The actual compositions were of $239 \mathrm{NPB}$ and 761 HAT-CN for the 1:3 sample (with HAT-CN molar fraction of $76.1 \%$, or $67.5 \%$ in weight), 897 and 103 for the $9: 1$ sample (10.3\%, 7\% in weight), and 1145 and 55 for the $29: 1$ sample $(4.6 \%, 3 \%$ in weight). The three systems were subsequently cooled and equilibrated at $300 \mathrm{~K}$, and subsequently at $200 \mathrm{~K}$ and $100 \mathrm{~K}$. At each temperature, equilibration was continued until no appreciable drift of the total energy was detected, compared to its thermal fluctuations: e.g. at $100 \mathrm{~K}$ energy fluctuations of about $0.02 \mathrm{kcal} \mathrm{mol}^{-1}$. Molecular geometries were then extracted from the equilibrated configurations and used for electronic structure calculations.

Ionization potentials IP $\mathrm{NPB}$ and electron affinities $\mathrm{EA}_{\mathrm{HAT}-\mathrm{CN}}^{\text {gas }}$ were computed at the gas-phase DFT level with the range-separated hybrid (RSH) functional $\omega \mathrm{B} 97 \mathrm{X}-\mathrm{D}^{45}$ along with the $6-311++\mathrm{G}(\mathrm{d}, \mathrm{p})$ basis set using the GAUSSIAN16 suite. ${ }^{46}$ These transport levels were evaluated as total energy differences between charged and neutral species, correcting for the inaccuracies of the FF in describing molecular geometries as follows. The gas-phase energy of a generic electronic state, is calculated as:

$$
\mathrm{E}\left(\mathbf{R}_{\mathrm{MD}}\right)=\mathrm{E}^{\mathrm{GW}}\left(\mathbf{R}_{\mathrm{X}}\right)+\left[\mathrm{E}^{\mathrm{DFT}}\left(\mathbf{R}_{\mathrm{MD}}\right)-\mathrm{E}^{\mathrm{DFT}}\left(\mathbf{R}_{\mathrm{X}}\right)\right]
$$

where $\mathbf{R}_{\mathrm{MD}}$ labels the geometry of a given molecule in the MD sample and $\mathbf{R}_{\mathrm{X}}$ is a reference geometry, taken from the bulk crystal structure of both species. In practice, the energy at the crystal geometry was calculated with the $G W$ method, which ensure accurate absolute values, and the energy deviations from it, as obtained from classical MD simulation geometries, were evaluated with DFT. Gas-phase $G W$ calculations were performed with the FIESTA code, starting from DFT calculations, 
based on the PBE0 functional, obtained with the ORCA package. ${ }^{47}$ A partial self-consistent scheme on the eigenvalues (evGW) was exploited, along with Gaussians basis set of the Dunning's correlation-consistent family (cc-pVXZ, where $X=2,3$ ). Then, quasi-particle energy levels were extrapolated to the complete basis set limit. ${ }^{14}$ The universal Weigend Coulomb fitting set of functions was used as auxiliary basis in the resolution of identity (RI-V) approach.

ME calculations ${ }^{12,13}$ were parametrized with ESP atomic charges and polarizability tensor calculated with DFT at the $\omega$ B97X-D/6-311++G(d,p) level. ESP charges were computed for all molecules in one equilibrated MD configuration, in neutral, positively (for NPB) and negatively (HAT-CN) charged states. The polarizability tensor was calculated at neutral NPB and HAT-CN reference geometries, and the polarizability of charged species was set equal to that of neutral ones. Polarization energies for holes $\left(\Delta^{+}\right)$and electrons $\left(\Delta^{-}\right)$were calculated for spherical clusters of increasing radius centered at the molecule of interest, and then extrapolated to the bulk, infinite radius limit. The solidstate energy levels for holes on NPB and electrons on HAT-CN can be expressed as: $I P_{N P B}=$ $I P_{N P B}^{\text {gas }}+\Delta^{+}$and $E A_{H A T-C N}=E A_{H A T-C N}^{g a s}+\Delta^{-}$. The energy of CT states was calculated for molecular NPB:HAT-CN pairs in close contact, which were selected from MD samples by applying $\mathrm{PBC}$ in the XY plane and including only molecules in the bulk region, i.e. excluding the molecules within $4 \mathrm{~nm}$ from the graphene substrate and vacuum interface in order to avoid empty spaces (in the spherical clusters) in the $\mathrm{z}$ direction. The photovoltaic gap is defined as $E_{G A P}=I P_{N P B}-E A_{H A T-C N}$. The optical gap is $E_{C T}=E_{G A P}+E_{B}$, where $E_{B}<0$ is the Coulomb exciton interaction between closely spaced electron and holes. The latter is calculated with self-consistent ME calculations as the screened interaction between hole and electron charge densities.

The effect of intramolecular structural relaxation upon charging was considered by calculating, separately for NPB and HAT-CN, the intramolecular reorganization energy $\lambda^{q}$, averaged on the charging and discharging processes:

$$
\lambda^{q}=\frac{\left|E^{q}\left(\boldsymbol{R}_{0}\right)-E^{q}\left(\boldsymbol{R}_{q}\right)\right|+\left|E^{0}\left(\boldsymbol{R}_{q}\right)-E^{0}\left(\boldsymbol{R}_{0}\right)\right|}{2}
$$

where $q$ stands for the charge on the molecule and $\boldsymbol{R}_{0}, \boldsymbol{R}_{q}$ indicate the equilibrium geometries of

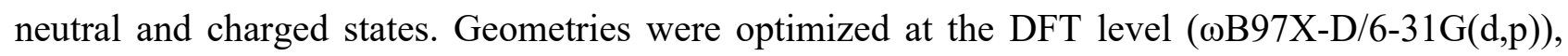
keeping the molecular soft degrees of freedom (dihedral angles) frozen, as to account only the contribution from high-frequency modes only ${ }^{48,49}$. This avoids double counting electron-vibration interactions, since the contribution of low-frequency vibrations was evaluated classically from MD simulations ( $\sigma_{\text {low }}^{x}$ in Tables 1 and 2). With this caveat, the total internal reorganization energy for CT formation / recombination is $\lambda_{\text {int }}=\lambda_{N P B}^{+}+\lambda_{H A T-C N}^{-}=262 \mathrm{meV}$, with $\lambda_{N P B}^{+}=184 \mathrm{meV}$ and $\lambda_{H A T-C N}^{-}=78 \mathrm{meV}$.

The conformational disorder was assessed with gas-phase DFT calculations performed at the MD geometries, quantifying the fluctuations of the NPB HOMO and the HAT-CN LUMO. The high- and low-frequency contributions to the dynamic conformational disorder were separated by means of a Fourier filter procedure applied to the time series of molecular orbital energies computed along the 
MD trajectories at $300 \mathrm{~K}$ (Figure S3 in SI). The low- (high-)frequency disorder $\sigma_{\text {low }}^{x}\left(\sigma_{\text {high }}^{x}\right)$ was evaluated as the standard deviation of the time series fluctuations (around the mean value of each molecule) after applying a low-pass (high-pass) filter to the original data (10 ps long trajectory sampled every $10 \mathrm{fs}$ ). The filter employed a rectangular function with energy cutoff equal to $\mathrm{k}_{\mathrm{B}} \mathrm{T}$ $\left(\sim 200 \mathrm{~cm}^{-1}\right)$. The variances of the frequency-filtered time series were averaged over $5 \mathrm{NPB}$ and 5 HAT-CN molecules to obtain the standard deviations in Table 3. Low- and high-frequency fluctuations were found to be uncorrelated.

Table 3: Standard deviations (in meV) of the intramolecular energy levels dynamic fluctuations along the MD trajectory, partitioned into the low- and high-frequency components disorder $\sigma_{\text {low }}^{x}$ and $\sigma_{\text {high }}^{x}$, with $\sigma_{d y n}^{x}=\sqrt{\sigma_{\text {low }}^{2 x}+\sigma_{\text {high }}^{2 x}}$. The samples are labelled according to the HAT-CN molar fraction and the MD simulation temperature.

\begin{tabular}{|l|l|l|l|l|l|l|}
\hline & \multicolumn{3}{|c|}{ NPB } & \multicolumn{3}{c|}{ HAT-CN } \\
\hline$\% / \mathrm{T}(\mathrm{K})$ & $\sigma_{\text {low }}^{I P}$ & $\sigma_{\text {high }}^{I P}$ & $\sigma_{\text {dyn }}^{I P}$ & $\sigma_{\text {low }}^{E A}$ & $\sigma_{\text {high }}^{\text {EA }}$ & $\sigma_{\text {dyn }}^{E A}$ \\
\hline $4.6 / 100$ & 59 & 40 & 71 & 11 & 39 & 40 \\
\hline $4.6 / 300$ & 87 & 67 & 110 & 19 & 60 & 63 \\
\hline $76.1 / 100$ & 68 & 39 & 78 & 14 & 36 & 38 \\
\hline $76.1 / 300$ & 102 & 60 & 118 & 19 & 54 & 57 \\
\hline
\end{tabular}

By filtering out the high-frequency contribution, the data in Table 3 allowed also the estimation of the static component of the total energetic disorder $\sigma_{\text {tot }}^{x}=\sqrt{\sigma_{\text {stat }}^{2^{x}}+\sigma_{\text {low }}^{2^{x}}}$, where $\sigma_{\text {tot }}^{x}$ was obtained as the full $\mathrm{IP}_{\mathrm{NPB}} / \mathrm{EA}_{\mathrm{HAT}-\mathrm{CN}} / \mathrm{E}_{\mathrm{GAP} / \mathrm{CT}}$ standard deviation for all NPB:HAT-CN pairs extracted from the MD configurations.

Non-radiative rates between the (first) CT state and the ground state were calculated with a perturbative approach with the semi-classical Marcus-Levich-Jortner expression:

$$
\kappa_{N R A D}=\frac{2 \pi}{\hbar} J^{2} \sqrt{\frac{1}{4 \pi \lambda_{e n v} k_{B} T}} \times \sum_{n}\left\{\exp \left(-S_{e f f}\right) \frac{S_{e f f}^{n}}{n !} \times \exp \left[-\frac{\left(-E_{C T}^{0,0}+\lambda_{\text {env }}+n \hbar \omega_{\text {eff }}\right)^{2}}{4 \lambda_{\text {env }} k_{B} T}\right]\right\}
$$

where $J$ is the electronic coupling between those states, computed with the Generalized MullikenHush (GMH) scheme (Equation 4$), \hbar$ is the reduced Planck's constant, $k_{B}$ is the Boltzmann constant, $T$ is the temperature in Kelvin, $\lambda_{\text {env }}$ is the environmental reorganization energy (see Table 2) computed from $\sigma_{\text {low }}$ as $\lambda_{e n v}=\frac{\sigma^{2} x}{2 k_{B} T}, \omega_{e f f}=0.15 \mathrm{eV}$ is the energy of an effective high-frequency intramolecular vibration (typically the C-C stretching), $S=\lambda_{\text {int }} /\left(\hbar \omega_{\text {eff }}\right)$ is the corresponding HuangRhys factor, and $\mathrm{E}_{\mathrm{CT}}^{0,0}=\mathrm{E}_{\mathrm{CT}}-\lambda_{\text {int }}$ is the GW/ME energy, where $\lambda_{\text {int }}$ is removed to avoid doublecounting with the term $\mathrm{n} \hbar \omega_{\text {eff }}$. 
The electronic couplings were computed for NPB:HAT-CN dimers in the $4.6 \%$ and $76.1 \%$ of HAT$\mathrm{CN}$ samples at $300 \mathrm{~K}$ at DFT/TD-DFT $\omega \mathrm{B} 97 \mathrm{X}-\mathrm{D} / 6-311 \mathrm{G}(\mathrm{d}, \mathrm{p})$ level of theory, and using a polarized continuum model (PCM), ${ }^{50}$ setting the scalar dielectric constant $\varepsilon=3.0$ or $\varepsilon=2.6$ for the sample at $4.6 \%$ or $76.1 \%$ of HAT-CN, respectively. The dielectric costant of the medium was obtained as the ratio between the unscreened interaction between an electron-hole pair $\left(\mathrm{V}_{\mathrm{eh}}\right)$ and the corresponding screened exciton binding energy $\left(\mathrm{E}_{\mathrm{B}}\right)$, both quantities accessible via ME calculations. The rangeseparation parameter $\omega^{51}$ was set to $0.09 \mathrm{Bohr}^{-1}$, a value which ensures, at it should be, a strong intermolecular CT character of the first excited state in NPB:HAT-CN dimer calculations. The electronic couplings in Equation 3 (see Figures S5 and S6 in SI) were calculated as ${ }^{52}$ :

$$
J=\frac{\vec{\mu}_{G S-C T} E_{C T}^{0,0}}{\sqrt{\Delta \mu_{G S-C T}^{2}+4 \vec{\mu}_{G S-C T}^{2}}}
$$

where $\vec{\mu}_{G S-C T}$ is the transition dipole moment, and $\Delta \mu_{G S-C T}$ the difference between the CT state permanent dipole moment and the ground state one.

The CT absorption band of a single NPB:HAT-CN dimer was calculated as a Frank-Condon progression:

$$
A b s(E)=\vec{\mu}_{G S-C T}^{2} \sum_{n=0}^{n_{\max }} \frac{e^{-S} S^{n}}{n !} \delta\left(E-E_{C T}^{0,0}-n \hbar \omega_{e f f}\right)
$$

where $\delta$ is the Dirac delta function. The absorption spectrum of the blend was computed as the superposition of CT bands of NPB:HAT-CN dimers in the sample, summing up the intensity of the individual vibronic transitions in bins of $100 \mathrm{meV}$. The CT band width was sourced from the energetic disorder of the sample and the convergence of the spectral shape was obtained with $n_{\max }=8$.

EQE measurements Samples for the EQE measurements were made on patterned ITO substrates (Colorado Concept Coatings). Substrates were cleaned by successive sonications in deionized water (15 min), acetone (10 $\mathrm{min})$, and isopropanol $(10 \mathrm{~min})$ at $40{ }^{\circ} \mathrm{C}$. Following the sonication step, the substrates were treated by oxygen plasma for $10 \mathrm{~min}$.

Solar cell samples for the EQE measurements were made by thermally co-evaporating $100 \mathrm{~nm}$ of NPB (Nichem) and HAT-CN (Nichem) in a thermal evaporator (Angstrom Engineering Inc.), under $\sim 10^{-7}$ torr base pressure, followed by $10 \mathrm{~nm}$ bathocuproine (BCP) (Lumtec) and $100 \mathrm{~nm}$ aluminum (Kurt J. Lesker Company) depositions. NPB and BCP were purified through thermal gradient sublimation, HAT-CN was used as purchased.

Temperature-dependent EQE spectra were measured by placing the sample inside a liquid $\mathrm{N}_{2}$ based cryostat (Janis VNF-100). The temperature was controlled by a LakeShore 335 Cryogenic Temperature Controller. Monochromatic light was generated using Newport TLS-300X, which was chopped at $390 \mathrm{~Hz}$ with an optical chopper. The short-circuited device photocurrent was amplified by a current preamplifier (SR570, Stanford Research Systems) and detected by a lock-in amplifier (SR830, Stanford Research Systems). The incoming photon quantities from the monochromatic light 
source were estimated using calibrated $\mathrm{Si}$ and Ge photodiodes (Newport corporation). The cryostat was cooled down to $110 \mathrm{~K}$ first and then brought up to the room temperature while taking the measurements. Room temperature EQE spectra before and after cooling did not show any sign of device degradation due to the temperature change.

In the framework of Marcus theory of electron transfer, a Gaussian function fitted to the low-energy edge of the EQE spectrum is commonly used as an approximation to the lowest energy CT absorption line-shape ${ }^{53}$, a technique that only considers vibronic broadening of the spectrum. However, Burke et al. ${ }^{35}$ demonstrated that, when a Gaussian CT energetic distribution is assumed with a peak $E_{C T}$ and standard distribution $\sigma_{\text {stat }}$, the overall $\mathrm{CT}$ absorption line-shape remains Gaussian with a shifted effective CT energy $\left(E_{C T(\text { exp.) }}\right)$. The modified absorption line-shape also demonstrates an effective reorganization energy $\left(\lambda_{(\text {exp. })}\right)$. These effective CT spectral quantities are related to $E_{C T}$ and reorganization energy $(\lambda)$ of the electron transfer process as:

$$
E_{C T(\exp .)}=E_{C T}-\frac{\sigma_{\text {stat }}^{2}}{2 k_{B} T} \quad \text { (6) } \quad \lambda_{(\text {exp. })}=\lambda+\frac{\sigma_{\text {stat }}^{2}}{2 k_{B} T}
$$

which can be extracted by fitting a Marcus type Gaussian line-shape to the low-energy EQE tail. The introduction of the static disorder in the $\mathrm{CT}$ absorption line-shape introduces a temperature dependence in the experimentally extracted CT state energy, which is clear from the $E_{C T(\text { exp.) }}$ and $\lambda_{(\text {exp.) }}$ values (see Figure S7a and S7b in SI) extracted from Figure 5a. A linear fitting to $E_{C T(\text { exp.) }}$ gives $\sigma_{\text {stat }}$ of $\sim 150 \mathrm{meV}$, responsible for the static broadening of the lowest energy CT EQE spectra. According to Burke et al.'s treatment of CT EQE spectra, the total variance of the CT EQE line-shape is:

$$
\sigma_{E Q E}^{2}=\sigma_{\text {stat }}^{2}+2 \lambda k_{B} T
$$

where $\sigma_{d y n}^{2}=2 \lambda k_{B} T$ is the contribution from the dynamic (vibrational) broadening of the CT spectra. The relative contribution of static disorder at a specific temperature could be defined as ${ }^{54}$ :

$$
D_{\text {stat }}(T)=\frac{\sigma_{\text {stat }}^{2}}{\sigma_{\text {stat }}^{2}+2 \lambda k_{B} T}
$$

The CT EQE lineshape, proposed by Burke et al..$^{35}$ considering a Gaussian energetic distribution of the CT states, is given as a function of incident photon energy $(\mathrm{E})$ :

$$
E Q E_{C T}(E) \propto \frac{1}{E \sqrt{2 \pi\left(\sigma_{\text {stat }}^{2}+2 \lambda k_{B} \mathrm{~T}\right)}} \exp \left(\frac{-\left(E_{C T}+\lambda-E\right)^{2}}{2 \sigma_{\text {stat }}^{2}+4 \lambda k_{B} T}\right)
$$

\section{ASSOCIATED CONTENT}

The Supporting information is available free of charge on the ... at DOI:

Distribution of NPB dihedrals at different temperatures; mixed NPB:HAT-CN radial distribution functions; time fluctuations and Fourier transform of orbital energies; average IP, EA, gap, CT energies and standard deviations at $100 \mathrm{~K}$; CT DOS at 100 and $300 \mathrm{~K}$; electronic couplings and non-radiative rates at $300 \mathrm{~K}$; fitting of effective lowest $\mathrm{CT}$ state and 
reorganization energies; and static $\left(\sigma_{\text {stat }}\right)$ and dynamic $\left(\sigma_{\text {dyn }}\right)$ disorder contribution to the CT EQE spectral broadening.

\section{CORRESPONDING AUTHOR}

*E-mail David.BELJONNE@umons.ac.be

\section{NOTES}

The authors declare no competing financial interest.

\section{ACKNOWLEDGMENTS}

This study received funding from the European Union's Horizon 2020 research and innovation program under the Marie Skłodowska-Curie Grant agreement No. 722651 (SEPOMO project) and from the European Union Horizon 2020 research and innovation programme under grant agreement no. 646176 (EXTMOS). L.M. thanks MIUR for financial support through PRIN Project 2015XJA9NT "Molecular Organization in Organic Thin Films via Computer Simulation of their Fabrication Processes". Computational resources were provided by the Consortium des Équipements de Calcul Intensif (CÉCI), funded by the Fonds de la Recherche Scientifique de Belgique (F.R.S.FNRS) under Grant No. 2.5020.11, as well as the Tier-1 supercomputer of the Fédération WallonieBruxelles, infrastructure funded by the Walloon Region under Grant Agreement No. 1117545. G.L., L.M., G.D. and D.B. gratefully thank Xavier Blase for fruitful discussions on many-body perturbation theory and for sharing the FIESTA code. D.B. is a FNRS Research Director. S.U.Z.K. and B.P.R. acknowledge the U.S. Department of Energy, Office of Basic Energy Sciences under Award No. DESC0012458.

\section{REFERENCES}

(1) Liu, Q.; Jiang, Y.; Jin, K.; Qin, J.; Xu, J.; Li, W.; Xiong, J.; Liu, J.; Xiao, Z.; Sun, K.; Yang, S.; Zhang, X.; Ding, L. 18\% Efficiency Organic Solar Cells. Sci. Bull. 2020, 65 (4), 272-275.

(2) Cui, Y.; Yao, H.; Zhang, J.; Xian, K.; Zhang, T.; Hong, L.; Wang, Y.; Xu, Y.; Ma, K.; An, C.; He, C.; Wei, Z.; Gao, F.; Hou, J. Single-Junction Organic Photovoltaic Cells with Approaching 18\% Efficiency. Adv. Mater. 2020, 1908205.

(3) Fusella, M. A.; Brigeman, A. N.; Welborn, M.; Purdum, G. E.; Yan, Y.; Schaller, R. D.; Lin, Y. L.; Loo, Y.-L.; Voorhis, T. Van; Giebink, N. C.; Rand, B. P. Band-like Charge Photogeneration at a Crystalline Organic Donor/Acceptor Interface. Adv. Energy Mater. 2018, 8 (9), 1701494.

(4) D’Avino, G.; Mothy, S.; Muccioli, L.; Zannoni, C.; Wang, L.; Cornil, J.; Beljonne, D.; Castet, F. Energetics of Electron-Hole Separation at P3HT/PCBM Heterojunctions. J. Phys. Chem. C 2013, 117 (25), 12981-12990.

(5) Castet, F.; D’Avino, G.; Muccioli, L.; Cornil, J.; Beljonne, D. Charge Separation Energetics at Organic Heterojunctions: On the Role of Structural and Electrostatic Disorder. Phys. Chem. Chem. Phys. 2014, 16 (38), 20279-20290.

(6) D'Avino, G.; Muccioli, L.; Olivier, Y.; Beljonne, D. Charge Separation and Recombination at Polymer-Fullerene Heterojunctions: Delocalization and Hybridization Effects. J. Phys. Chem. Lett. 2016, 7 (3), 536-540.

(7) Lin, Y. L.; Fusella, M. A.; Rand, B. P. The Impact of Local Morphology on Organic Donor/Acceptor Charge Transfer States. Adv. Energy Mater. 2018, 8 (28), 1702816.

(8) Vandewal, K.; Albrecht, S.; Hoke, E. T.; Graham, K. R.; Widmer, J.; Douglas, J. D.; Schubert, M.; Mateker, W. R.; Bloking, J. T.; Burkhard, G. F.; Sellinger, A.; Fréchet, J. M. 
J.; Amassian, A.; Riede, M. K.; McGehee, M. D.; Neher, D.; Salleo, A. Efficient Charge Generation by Relaxed Charge-Transfer States at Organic Interfaces. Nat. Mater. 2014, 13 (1), 63-68.

(9) Panhans, M.; Hutsch, S.; Benduhn, J.; Schellhammer, K. S.; Nikolis, V. C.; Vangerven, T.; Vandewal, K.; Ortmann, F. Molecular Vibrations Reduce the Maximum Achievable Photovoltage in Organic Solar Cells. Nat. Commun. 2020, 11 (1), 1-10.

(10) Lin, Y. L.; Fusella, M. A.; Kozlov, O. V.; Lin, X.; Kahn, A.; Pshenichnikov, M. S.; Rand, B. P. Morphological Tuning of the Energetics in Singlet Fission Organic Solar Cells. $A d v$. Funct. Mater. 2016, 26 (35), 6489-6494.

(11) Khan, S. U. Z.; Londi, G.; Liu, X.; Fusella, M. A.; D’Avino, G.; Muccioli, L.; Brigeman, A. N.; Niesen, B.; Yang, T. C. J.; Olivier, Y.; Dull, J. T.; Giebink, N. C.; Beljonne, D.; Rand, B. P. Multiple Charge Transfer States in Donor-Acceptor Heterojunctions with Large Frontier Orbital Energy Offsets. Chem. Mater. 2019, 31 (17), 6808-6817.

(12) D’Avino, G.; Muccioli, L.; Zannoni, C.; Beljonne, D.; Soos, Z. G.; D’Avino, G.; Muccioli, L.; Zannoni, C.; Beljonne, D.; Soos, Z. G.; D’Avino, G.; Muccioli, L.; Zannoni, C.;

Beljonne, D.; Soos, Z. G. Electronic Polarization in Organic Crystals: A Comparative Study of Induced Dipoles and Intramolecular Charge Redistribution Schemes. J. Chem. Theory Comput. 2014, 10 (11), 4959-4971.

(13) D’Avino, G.; Muccioli, L.; Castet, F.; Poelking, C.; Andrienko, D.; Soos, Z. G.; Cornil, J.; Beljonne, D. Electrostatic Phenomena in Organic Semiconductors: Fundamentals and Implications for Photovoltaics. J. Phys. Condens. Matter 2016, 28 (43).

(14) Li, J.; Duchemin, I.; Roscioni, O. M.; Friederich, P.; Anderson, M.; Da Como, E.; KociokKöhn, G.; Wenzel, W.; Zannoni, C.; Beljonne, D.; Blase, X.; D’Avino, G. Host Dependence of the Electron Affinity of Molecular Dopants. Mater. Horizons 2019, 6 (1), 107-114.

(15) Muccioli, L.; D’Avino, G.; Zannoni, C. Simulation of Vapor-Phase Deposition and Growth of a Pentacene Thin Film on C60 (001). Adv. Mater. 2011, 23 (39), 4532-4536.

(16) D'Avino, G.; Muccioli, L.; Zannoni, C. From Chiral Islands to Smectic Layers: A Computational Journey Across Sexithiophene Morphologies on C 60. Adv. Funct. Mater. 2015, 25 (13), 1985-1995.

(17) Tonnelé, C.; Stroet, M.; Caron, B.; Clulow, A. J.; Nagiri, R. C. R.; Malde, A. K.; Burn, P. L.; Gentle, I. R.; Mark, A. E.; Powell, B. J. Elucidating the Spatial Arrangement of Emitter Molecules in Organic Light-Emitting Diode Films. Angew. Chemie Int. Ed. 2017, 56 (29), 8402-8406.

(18) Roscioni, O. M.; D’Avino, G.; Muccioli, L.; Zannoni, C. Pentacene Crystal Growth on Silica and Layer-Dependent Step-Edge Barrier from Atomistic Simulations. J. Phys. Chem. Lett. 2018, 9 (23), 6900-6906.

(19) Lee, T.; Sanzogni, A.; Zhangzhou, N.; Burn, P. L.; Mark, A. E. Morphology of a Bulk Heterojunction Photovoltaic Cell with Low Donor Concentration. ACS Appl. Mater. Interfaces 2018, 10 (38), 32413-32419.

(20) Schwarze, M.; Tress, W.; Beyer, B.; Gao, F.; Scholz, R.; Poelking, C.; Ortstein, K.; Günther, A. A.; Kasemann, D.; Andrienko, D.; Leo, K. Band Structure Engineering in Organic Semiconductors. Science (80-. ). 2016, 352 (6292), 1446-1449.

(21) Graham, K. R.; Ndjawa, G. O. N.; Conron, S. M.; Munir, R.; Vandewal, K.; Chen, J. J.; Sweetnam, S.; Thompson, M. E.; Salleo, A.; McGehee, M. D.; Amassian, A. The Roles of Structural Order and Intermolecular Interactions in Determining Ionization Energies and Charge-Transfer State Energies in Organic Semiconductors. Adv. Energy Mater. 2016, 6 (22), 1601211.

(22) Privitera, A.; Londi, G.; Riede, M.; D’Avino, G.; Beljonne, D. Molecular Quadrupole Moments Promote Ground-State Charge Generation in Doped Organic Semiconductors. Adv. Funct. Mater. 2020, $n / a(\mathrm{n} / \mathrm{a}), 2004600$.

(23) Hill, I. G.; Kahn, A. Organic Semiconductor Heterointerfaces Containing Bathocuproine. $J$. 
Appl. Phys. 1999, 86 (8), 4515-4519.

(24) Amsalem, P.; Wilke, A.; Frisch, J.; Niederhausen, J.; Vollmer, A.; Rieger, R.; Müllen, K.; Rabe, J. P.; Koch, N. Interlayer Molecular Diffusion and Thermodynamic Equilibrium in Organic Heterostructures on a Metal Electrode. J. Appl. Phys. 2011, 110 (11), 113709.

(25) Yoshida, H.; Yoshizaki, K. Electron Affinities of Organic Materials Used for Organic LightEmitting Diodes: A Low-Energy Inverse Photoemission Study. Org. Electron. 2015, 20, 24 30.

(26) Falkenberg, C.; Olthof, S.; Rieger, R.; Baumgarten, M.; Muellen, K.; Leo, K.; Riede, M. The Role of Energy Level Matching in Organic Solar Cells- Hexaazatriphenylene Hexacarbonitrile as Transparent Electron Transport Material. Sol. Energy Mater. Sol. Cells 2011, 95 (3), 927-932.

(27) Tummala, N. R.; Zheng, Z.; Aziz, S. G.; Coropceanu, V.; Brédas, J.-L. Static and Dynamic Energetic Disorders in the C60, PC61BM, C70, and PC71BM Fullerenes. J. Phys. Chem. Lett. 2015, 6 (18), 3657-3662.

(28) D’Avino, G.; Olivier, Y.; Muccioli, L.; Beljonne, D. Do Charges Delocalize over Multiple Molecules in Fullerene Derivatives? J. Mater. Chem. C 2016, 4 (17), 3747-3756.

(29) de Silva, P.; Van Voorhis, T. QM/MM Study of Static and Dynamic Energetic Disorder in the Emission Layer of an Organic Light-Emitting Diode. J. Phys. Chem. Lett. 2018, 9 (6), 1329-1334.

(30) Mikhnenko, O. V.; Blom, P. W. M.; Nguyen, T. Q. Exciton Diffusion in Organic Semiconductors. Energy and Environmental Science. 2015, pp 1867-1888.

(31) Jacobs, I. E.; Moulé, A. J. Controlling Molecular Doping in Organic Semiconductors. Adv. Mater. 2017, 29 (42), 1703063.

(32) Zhang, L.; Zu, F.-S.; Deng, Y.-L.; Igbari, F.; Wang, Z.-K.; Liao, L.-S. Origin of Enhanced Hole Injection in Organic Light-Emitting Diodes with an Electron-Acceptor Doping Layer: P-Type Doping or Interfacial Diffusion? ACS Appl. Mater. Interfaces 2015, 7 (22), 11965 11971.

(33) Tvingstedt, K.; Benduhn, J.; Vandewal, K. Temperature Dependence of the Spectral LineWidth of Charge-Transfer State Emission in Organic Solar Cells; Static vs. Dynamic Disorder. Mater. Horizons 2020, 7 (7), 1888.

(34) Baranovskii, S. D. Theoretical Description of Charge Transport in Disordered Organic Semiconductors. Phys. status solidi 2014, 251 (3), 487-525.

(35) Burke, T. M.; Sweetnam, S.; Vandewal, K.; McGehee, M. D. Beyond Langevin Recombination: How Equilibrium Between Free Carriers and Charge Transfer States Determines the Open-Circuit Voltage of Organic Solar Cells. Adv. Energy Mater. 2015, 5 (11), 1500123.

(36) J. Benduhn, Ph.D. thesis, TU Dresden, 2019. No Title.

(37) Azzouzi, M.; Yan, J.; Kirchartz, T.; Liu, K.; Wang, J.; Wu, H.; Nelson, J. Nonradiative Energy Losses in Bulk-Heterojunction Organic Photovoltaics. Phys. Rev. X 2018, 8 (3), 031055.

(38) Bixon, M.; Jortner, J.; Cortes, J.; Heitele, H.; Michel-Beyerle, M. E. Energy Gap Law for Nonradiative and Radiative Charge Transfer in Isolated and in Solvated Supermolecules. $J$. Phys. Chem. 1994, 98 (30), 7289-7299.

(39) Benduhn, J.; Tvingstedt, K.; Piersimoni, F.; Ullbrich, S.; Fan, Y.; Tropiano, M.; McGarry, K. A.; Zeika, O.; Riede, M. K.; Douglas, C. J.; Barlow, S.; Marder, S. R.; Neher, D.; Spoltore, D.; Vandewal, K. Intrinsic Non-Radiative Voltage Losses in Fullerene-Based Organic Solar Cells. Nat. Energy 2017, 2 (6), 1-6.

(40) Kahle, F. J.; Rudnick, A.; Bässler, H.; Köhler, A. How to Interpret Absorption and Fluorescence Spectra of Charge Transfer States in an Organic Solar Cell. Mater. Horizons 2018, 5 (5), 837-848.

(41) Phillips, J. C.; Braun, R.; Wang, W.; Gumbart, J.; Tajkhorshid, E.; Villa, E.; Chipot, C.; 
Skeel, R. D.; Kalé, L.; Schulten, K. Scalable Molecular Dynamics with NAMD. J. Comput. Chem. 2005, 26 (16), 1781-1802.

(42) Moral, M.; Son, W.-J.; Sancho-García, J. C.; Olivier, Y.; Muccioli, L. Cost-Effective Force Field Tailored for Solid-Phase Simulations of OLED Materials. J. Chem. Theory Comput. 2015, 11, 3383-3392.

(43) Szalay, P. S.; Galán-Mascarós, J. R.; Clérac, R.; Dunbar, K. R. HAT(CN)6: A New Building Block for Molecule-Based Magnetic Materials. Synth. Met. 2001, 122 (3), 535-542.

(44) Steele, W. A. The Interaction of Rare Gas Atoms with Graphitized Carbon Black. J. Phys. Chem. 1978, 82 (7), 817-821.

(45) Lin, Y. S.; Li, G. De; Mao, S. P.; Chai, J. Da. Long-Range Corrected Hybrid Density Functionals with Improved Dispersion Corrections. J. Chem. Theory Comput. 2013, 9 (1), 263-272.

(46) Frisch, M. J.; Trucks, G. W.; Schlegel, H. B.; Scuseria, G. E.; Robb, M. A.; Cheeseman, J. R.; Scalmani, G.; Barone, V.; Petersson, G. A.; Nakatsuji, H.; Li, X.; Caricato, M.; Marenich, A. V; Bloino, J.; Janesko, B. G.; Gomperts, R.; Mennucci, B.; Hratchian, H. P.; Ortiz, J. V; Izmaylov, A. F.; Sonnenberg, J. L.; Williams-Young, D.; Ding, F.; Lipparini, F.; Egidi, F.; Goings, J.; Peng, B.; Petrone, A.; Henderson, T.; Ranasinghe, D.; Zakrzewski, V. G.; Gao, J.; Rega, N.; Zheng, G.; Liang, W.; Hada, M.; Ehara, M.; Toyota, K.; Fukuda, R.; Hasegawa, J.; Ishida, M.; Nakajima, T.; Honda, Y.; Kitao, O.; Nakai, H.; Vreven, T.; Throssell, K.; Montgomery Jr., J. A.; Peralta, J. E.; Ogliaro, F.; Bearpark, M. J.; Heyd, J. J.; Brothers, E. N.; Kudin, K. N.; Staroverov, V. N.; Keith, T. A.; Kobayashi, R.; Normand, J.;

Raghavachari, K.; Rendell, A. P.; Burant, J. C.; Iyengar, S. S.; Tomasi, J.; Cossi, M.; Millam, J. M.; Klene, M.; Adamo, C.; Cammi, R.; Ochterski, J. W.; Martin, R. L.; Morokuma, K.; Farkas, O.; Foresman, J. B.; Fox, D. J. Gaussian 16 Revision C.01. Gaussian Inc. Wallingford CT 2016.

(47) Neese, F. Software Update: The ORCA Program System, Version 4.0. WIREs Comput. Mol. Sci. 2018, 8 (1).

(48) Londi, G.; Dilmurat, R.; D’Avino, G.; Lemaur, V.; Olivier, Y.; Beljonne, D. Comprehensive Modelling Study of Singlet Exciton Diffusion in Donor-Acceptor Dyads: When Small Changes in Chemical Structure Matter. Phys. Chem. Chem. Phys. 2019, 21 (45), 2502325034.

(49) Marqués, P. S.; Andrés Castán, J. M.; Raul, B. A. L.; Londi, G.; Ramirez, I.; Pshenichnikov, M. S.; Beljonne, D.; Walzer, K.; Blais, M.; Allain, M.; Cabanetos, C.; Blanchard, P. Triphenylamine/Tetracyanobutadiene-Based $\pi$-Conjugated Push-Pull Molecules End-Capped with Arene Platforms: Synthesis, Photophysics, and Photovoltaic Response. Chem. - A Eur. J. 2020, $n / a(\mathrm{n} / \mathrm{a})$.

(50) Tomasi, J.; Mennucci, B.; Cammi, R. Quantum Mechanical Continuum Solvation Models. Chem. Rev. 2005, 105 (8), 2999-3094.

(51) Brémond, É.; Pérez-Jiménez, Á. J.; Sancho-García, J. C.; Adamo, C. Range-Separated Hybrid Density Functionals Made Simple. J. Chem. Phys. 2019, 150 (20), 201102.

(52) Cave, R. J.; Newton, M. D. Generalization of the Mulliken-Hush Treatment for the Calculation of Electron Transfer Matrix Elements. Chem. Phys. Lett. 1996, 249 (1-2), 15-19.

(53) Vandewal, K.; Tvingstedt, K.; Gadisa, A.; Inganäs, O.; Manca, J. V. Relating the OpenCircuit Voltage to Interface Molecular Properties of Donor:Acceptor Bulk Heterojunction Solar Cells. Phys. Rev. B 2010, 81 (12), 125204.

(54) Linderl, T.; Zechel, T.; Hofmann, A.; Sato, T.; Shimizu, K.; Ishii, H.; Brütting, W. Crystalline versus Amorphous Donor-Acceptor Blends: Influence of Layer Morphology on the Charge-Transfer Density of States. Phys. Rev. Appl. 2020, 13 (2), 24061. 